ROM2F/2005/02

\title{
The open story of the magnetic fluxes
}

\author{
M. Bianchi and E. Trevigne
}

Dipartimento di Fisica and Sezione INFN

Università di Roma "Tor Vergata"

00133 Rome, Italy

Massimo.Bianchi,Elisa. Trevigne@roma2.infn.it

\begin{abstract}
We discuss the effects of oblique internal magnetic fields on the spectrum of type I superstrings compactified on tori. In particular we derive general formulae for the magnetic shifts and multiplicities of open strings connecting D9-branes with arbitrary magnetic fluxes. We discuss the flux induced potential and offer an interpretation of the stabilization of R-R moduli associated to deformations of the complex structure of $\mathbf{T}^{6}$ in terms of non-derivative mixings with NS-NS moduli. Finally we briefly comment on how to extract other low energy couplings and generalize our results to toroidal orbifolds and other configurations governed by rational conformal field theories on the worldsheet.
\end{abstract}

\section{Introduction}

Constant electromagnetic fields coupled to the ends of open strings lead to many interesting effects that can be treated exactly in string theory [1, 2, 3, 4, 5] and have far reaching consequences in phenomenologically viable vacuum configurations [6, 7, 8, 9, 10, 11, 12. The presence of constant abelian magnetic fields changes the open string boundary conditions in such a way that the oscillator modes of charged strings get shifted and the zero modes of neutral strings (KK momenta and windings) get reshuffled [13, 14, 15].

So far most of the analyses focussed on "parallel" magnetic fields $H_{a}$ on "factorizable" tori $\mathbf{T}^{d}=\mathbf{T}^{2} \times \ldots \mathbf{T}^{2}$ or toroidal orbifolds that can be T-dualized to a single kind of branes intersecting at angles, for reviews see [16, 17, 18, 19]. The boundary conditions are coded in the mutually commuting $d \times d$ orthogonal matrices

$$
R_{a}=\left(\mathbb{1}-H_{a}\right)\left(\mathbb{1}+H_{a}\right)^{-1} .
$$

However, as shown in 20] for a toroidal compactification of type I strings to $D=4$, properly turning on oblique but yet abelian magnetic fields, such that 
$\left[R_{a}, R_{b}\right] \neq 0$, in "non-factorizable" subtori with common directions may lead to closed string moduli stabilization while keeping some residual supersymmetry. These configurations are T-dual to bound states of several different kinds of intersecting D-branes [21, 22, 23].

Here, we present a detailed analysis of the resulting open string spectrum and discuss the resulting potential for the closed string moduli fields, as well as their mixing and stabilization. In order to fix our notation and describe some of the relevant issues in a simpler setting we start by reviewing the very familiar case of a 2-dimensional torus in section 2 and the less familiar case of a 3-dimensional torus, where oblique magnetic fields first show up, in section [3. In both these cases, however, turning on magnetic fields breaks all supersymmetries and prevents the existence of any stable configuration at finite internal volume even if one introduces exotic $\Omega$-planes. We discuss the more interesting case of a 4-dimensional torus in section 4 where we derive a very general formula for the mode shifts in the presence of arbitrary magnetic fields and argue that all closed string moduli, except the overall volume and the dilaton, can be stabilized in a supersymmetric fashion. Scale invariance of the (anti) self-duality conditions should be held responsible for the arbitrariness of the overall volume. In section 5, we sketch how to embed these neat results into a 5-dimensional torus. All this is meant to be propaedeutic to the analysis of the phenomenologically relevant case of a 6-dimensional torus studied in section 6. After some general considerations, we restrict our attention to supersymmetric configurations and describe how to compute in full generality the magnetic shifts for oblique yet supersymmetry preserving magnetic fields. We also clarify the mechanism of stabilization of $\mathrm{R}-\mathrm{R}$ moduli associated to deformations of the complex structure in terms of non-derivative mixings with NS-NS moduli. To this end we adapt and generalize the closed string scattering amplitudes on D-branes computed in 24, 25] to our case and derive similar formulae for scattering on $\Omega$-planes [26]. We present our conclusions and perspectives for future investigation in section [7. In particular, we sketch how to extract tree-level and one-loop gauge couplings from (simple modifications of) the results in the previous sections. We also discuss some generalizations to toroidal orbifolds and to vacuum configurations described by rational conformal field theories on the worldsheet. Very much as in [27], we argue that magnetic fluxes lead to twisted representations of the relevant chiral algebra. 


\section{Two-dimensional magnetized torus, yet again}

A magnetic field on $\mathbf{T}^{2}$ is necessarily proportional to the volume (Kähler) form $J=d x^{1} d x^{2}=d \bar{z} d z / 2 i$. In complex coordinates, $F=F_{12} d x^{1} d x^{2}=i f d z d \bar{z} / 2$, and the corresponding magnetic rotation matrix reads

$$
R=(\mathbb{1}-F)(\mathbb{1}+F)^{-1}=\operatorname{diag}\left(e^{2 i \beta}, e^{-2 i \beta}\right)
$$

where

$$
\tan (\beta)=f \quad \text { so that } \quad \tan (2 \beta)=\frac{2 f}{1-f^{2}} \quad .
$$

In a real basis, one would have instead

$$
R=\left(\begin{array}{cc}
\frac{1-f^{2}}{1+f^{2}} & -\frac{2 f}{1+f^{2}} \\
\frac{2 f}{1+f^{2}} & \frac{1-f^{2}}{1+f^{2}}
\end{array}\right) .
$$

Consistency at the quantum level requires Dirac flux quantization

$$
f=\frac{m}{n} \frac{\alpha^{\prime}}{r_{1} r_{2}}
$$

where the magnetic monopole number $(m)$ and the "wrapping" number $(n)$ are integers, in the absence of a (quantized) NS-NS antisymmetric tensor background [28, 29, 30]. Otherwise $m$ gets shifted by $B n[13,14,15]$.

Thanks to the abelian nature of the rotation group in two dimensions, when several abelian magnetic fields are present the magnetic shifts of the modes of open strings connecting the stacks $a$ (with flux $f_{a}$ ) and $b$ (with flux $f_{b}$ ) are given by [2, 31, 32, 33, 4, 3]

$$
\epsilon_{a b}=\frac{\beta_{a b}}{\pi}=\frac{1}{\pi}\left[q_{a} \arctan \left(f_{a}\right)+q_{b} \arctan \left(f_{b}\right)\right]
$$

where $q_{a}, q_{b}= \pm 1$ take care of possible images under orientation reversal, i.e. worldsheet parity $\Omega$ [13]. Let us stress that the magnetic rotation

$$
R_{a b}=R^{q_{a}}\left(f_{a}\right) R^{q_{b}}\left(f_{b}\right)=R\left(q_{a} f_{a}+q_{b} f_{b}\right)=R\left(2 \beta_{a b}\right)
$$

appearing in the boundary state [34, acts by twice the amount determining the magnetic shift, $\beta_{a b}=\pi \epsilon_{a b}$.

In order to properly count the multiplicities of charged strings, one has to rescale $N_{a}$ to $\hat{N}_{a}=N_{a} / n_{a}$ as a consequence of the non-trivial wrapping. In general, the target space coordinates $X^{i}$ do not necessarily coincide with 
the world-volume coordinates $\sigma^{\alpha}$ but are rather linear functions of the latter. The jacobian matrix $W_{\alpha}{ }^{i}=\partial X^{i} / \partial \sigma^{\alpha}$ of the linear transformation mapping the world-volume $\Sigma^{d}$ onto the target space $\mathbf{T}^{d}$ must have integer entries. As a result, $W=\operatorname{det}\left(W_{\alpha}{ }^{i}\right)=n$, is an integer, e.g. $W=1$ for the trivial wrapping $X^{i}=\delta_{\alpha}^{i} \sigma^{\alpha}$. The induced world-volume metric is thus $\mathcal{G}_{\alpha \beta}=W_{\alpha}{ }^{i} W_{\beta}{ }^{j} G_{i j}$. Clearly there is a separate wrapping matrix per each stack of (magnetized) branes $W_{\alpha(a)}^{i}$ labeled by the index $a$ and $n_{a}=\operatorname{det}\left(W_{a}\right)$.

Open strings with $q_{b}=-q_{a}$ and $a \neq b$, belong to the representation $\left(\hat{N}_{a}, \hat{N}_{b}^{*}\right)$. For $q_{a}=q_{b}$, which is possible only in the unoriented case, one gets $\left(\hat{N}_{a}, \hat{N}_{b}\right)$. In both cases the degeneracy of the Landau levels

$$
I_{a b}=q_{a} m_{a} n_{b}+q_{b} m_{b} n_{a}
$$

coincides with the first Chern number of the abelian gauge bundle. In the superstring case, $I_{a b}$ counts the number of charged massless fermions, but the spectrum contains open string tachyons whenever $f_{a} \neq 0$ for some $a$.

Neutral strings starting and ending on the same stack $a=b$ of branes and thus with $q_{b}=-q_{a}$ have integer modes but carry "rescaled" KK momenta $p_{i}=k_{i} / n r_{i} \sqrt{1+f^{2}}$ with $i=1,2$. In the unoriented case, doubly charged strings, that start on a stack $a$ and end on its image $b=\tilde{a}$ under $\Omega$ with $q_{\tilde{a}}=q_{a}$ are counted by the first Chern number of the (anti) symmetric tensor gauge bundle.

T-duality along the $y=x^{2}$ axis maps D2-branes filling $\mathbf{T}^{2}$ into D1-branes wrapped around a direction forming an angle $\beta=\arctan \left(m \widetilde{R}_{2} / n R_{1}\right)$ with the $x=x^{1}$ axis 35. The magnetic shifts (up to a factor of $\pi$ ) are T-dual to the intersection angles. In the T-dual picture, $I_{a b}$ counts the number of "algebraic" intersections [19, while the magnetically rescaled momenta map to momenta along the non-trivially wrapped D1-branes and windings in the orthogonal direction 17, 14.

Very much as for the heterotic string on twisted tori [36], internal magnetic fluxes can generate a potential for the closed string NS-NS and R-R moduli, $G_{i j}$ and $C_{i j}$, as well as for the dilaton $\phi$ and the open string Wilson line moduli $A_{i}^{a}$ 37, 38, 39]. The crucial difference is that for (unoriented) open strings the potential appears at half closed string loop order. The Born-Infeld contribution of the D-branes (disk) and the (negative) tension contribution of the $\Omega$-plane (projective plane) [40, 41] combine to

$$
\left.V\left(\phi, G_{i j} ; m_{a}, n_{a}\right)=\tau e^{-\phi}\left(2 \sum_{a} N_{a} \sqrt{\operatorname{det}\left(\mathcal{G}_{a}+\mathcal{F}_{a}\right.}\right) \mp 32 \sqrt{\operatorname{det}(G)}\right)
$$


where $\mathcal{G}_{\alpha \beta}^{a}$, as discussed above, is the induced metric on the $a^{\text {th }}$ stack of branes, $\mathcal{F}_{\alpha \beta}^{a}$ is the world-volume magnetic field

$$
\mathcal{F}_{\alpha \beta}^{a}=\partial_{\alpha} A_{\beta}^{a}-\partial_{\beta} A_{\alpha}^{a}=W_{\alpha}^{(a) i} W_{\beta}^{(a) j} F_{i j}^{a},
$$

and $\tau$ is the constant (moduli independent) part of the brane tension. The factor of 2 takes care of image branes and we have allowed for exotic $\Omega$-planes with positive tension. Neglecting open string moduli and the WZ coupling to the R-R moduli, $V$ turns out to depend only on the positive-definite overall volume $\omega=\sqrt{\operatorname{det}(G)}$ and the dilaton $\phi$ but not on the complex structure moduli. Independently of the sign of the $\Omega$-plane tension,

$$
V\left(\phi, \omega ; m_{a}, n_{a}\right)=\tau e^{-\phi}\left(2 \sum_{a} N_{a} \sqrt{n_{a}^{2} \omega^{2}+m_{a}^{2}} \mp 32|\omega|\right)
$$

displays a runaway behavior to infinity for $\phi$. $\omega$ runs to infinite, respectively zero, value for standard, respectively, exotic (anti) $\Omega$-planes. The R-R tadpole condition, $2 \sum_{a} n_{a} N_{a}= \pm 32$, can be satisfied for standard $\Omega$-planes and exotic $\bar{\Omega}$-planes 29, 42 .

We will not dwelve any further on the issue of moduli stabilization on $\mathbf{T}^{2}$ since magnetic fluxes break supersymmetry anyway and generate tachyons in the open superstring spectrum. In passing, the situation does not improve very much for compactifications on product tori $\times_{i} \mathbf{T}_{(i)}^{2}$ and orbifolds thereof, which have been the main subject of investigation so far. The above formulae for the magnetic shifts, multiplicities and zero-modes can be straightforwardly generalized. Same for the Born-Infeld and WZ couplings [40, 41]. However the only NS-NS moduli that appear in the potential are the volume moduli $\omega_{i}$ and they cannot be stabilized.

\section{Three-dimensional magnetized torus}

In three dimensions a magnetic field has three independent components $F=$ $F_{x y} d x d y+F_{y z} d y d z+F_{z x} d z d x$. Any proper rotation has at least one unit eigenvalue and two complex conjugate ones of unit norm. By elementary physics the unmagnetized direction points along the magnetic field $\vec{F}=\left(F_{y z}, F_{z x}, F_{x y}\right)$.

Setting $f=|\vec{F}|$, the modes of the coordinates orthogonal to $\vec{F}$ of a singly charged open string, starting on a magnetic brane and ending on an unmagnetized one, are shifted by

$$
\epsilon=\frac{\beta}{\pi} \quad \text { with } \quad \beta=\arctan (f)
$$


The situation is slightly more involved for a string starting on a magnetized brane labeled by $a$ and ending on another one labeled by $b$. As anticipated in the introduction and suggested by boundary state considerations 34, one has to diagonalize the rotation matrix $R_{a b}=R_{a}^{q_{a}} R_{b}^{q_{b}}$, where $R_{a},\left(R_{b}\right)$ is a rotation around $\vec{F}_{a}\left(\vec{F}_{b}\right)$ by an angle $2 \beta_{a}\left(2 \beta_{b}\right)$. The eigenvector corresponding to the unit eigenvalue identifies the unmagnetized direction $\vec{u}_{a b}$, while the two orthogonal directions generically suffer a non-trivial action of $R_{a b}$. For practical reasons, instead of working in the 3-dimensional vector representation of $S O(3) \approx S U(2)$, it turns out to be much more rewarding to work in the spin $1 / 2$ representation and exploit the remarkable properties of Pauli matrices. A magnetic rotation can be written as

$$
R_{(1 / 2)}^{q}=\cos (\beta)-i q \hat{F} \cdot \sigma \sin (\beta)
$$

where $\hat{F}=\vec{F} /|\vec{F}|$ is the unit vector in the direction of the magnetic field. Let us stress once more that the argument of the trigonometric functions is correctly $\beta=2 \beta / 2$. Denoting for short $\cos \left(\beta_{a / b}\right)$, respectively $\sin \left(\beta_{a / b}\right)$, by $c_{a / b}$, respectively $s_{a / b}$, and combining the two rotations yields

$$
R_{a b}=\left(c_{a} c_{b}-q_{a} q_{b} s_{a} s_{b} \hat{F}_{a} \cdot \hat{F}_{b}\right)-i\left(q_{a} s_{a} c_{b} \hat{F}_{a}+q_{b} c_{a} s_{b} \hat{F}_{b}+q_{a} q_{b} s_{a} s_{b} \hat{F}_{a} \times \hat{F}_{b}\right) \cdot \sigma
$$

that exposes the 'unmagnetized' direction

$$
\vec{u}_{a b}=q_{a} s_{a} c_{b} \hat{F}_{a}+q_{b} c_{a} s_{b} \hat{F}_{b}+q_{a} q_{b} s_{a} s_{b} \hat{F}_{a} \times \hat{F}_{b}
$$

and determines the magnetic shifts $\epsilon_{a b}=\beta_{a b} / \pi$ via

$$
\cos \left(\beta_{a b}\right)=c_{a} c_{b}-q_{a} q_{b} s_{a} s_{b} \hat{F}_{a} \cdot \hat{F}_{b} .
$$

Though obvious, let us stress once more that $\beta_{a b} \neq \beta_{a} \pm \beta_{b}$ in general, contrarily to the two-dimensional as bad as any factorizable case. Another amusing feature is the orientation of the 'unmagnetized' direction $\vec{u}_{a b}$ with respect to the generators of the fundamental cell. Due to Dirac quantization condition on $F_{a}$, in each sector of the spectrum one can redefine the fundamental cell and choose $\vec{u}_{a b}$ to be one of the generators thereof. The generalized KK momenta carried by neutral strings turn out then to be rather involved combinations of momenta and windings. These are not so surprising features of the non-abelian structure of the rotation group.

Unfortunately, there is no non-trivial way to preserve supersymmetry and, as in the two dimensional case, one can neither avoid tachyons when non-trivial magnetic fields are turned on, nor stabilize the vacuum perturbatively. 


\section{Four-dimensional magnetized torus}

Magnetized four-dimensional tori display some remarkable features. This is the lowest dimensional case where non-trivial supersymmetric configurations first appear. Moreover thanks to the non-simplicity of the rotation group, $S O(4)=S U(2)_{L} \times S U(2)_{R}$, one can compactly analyze the problem for arbitrary magnetic fields. To this end it is very convenient to choose a basis for the six constant two-forms comprising three self-dual $\omega^{a}$ and as many anti self-dual $\bar{\omega}^{a}$ two-forms and decompose $F$ according to

$$
F=\frac{1}{2} F_{i j} d x^{i} d x^{j}=F^{+}+F^{-}=f_{r} \omega^{r}+\bar{f}_{r} \bar{\omega}^{r}
$$

This can be rather explicitly achieved, for instance, by using 't Hooft symbols $\eta_{m n}^{a}$ and $\bar{\eta}_{i j}^{r}$, with $r=1,2,3$ and $i, j=1, \ldots 4$, and setting $\omega^{r}=\eta_{i j}^{r} d x^{i} d x^{j} / 2$ and $\bar{\omega}^{r}=\bar{\eta}_{i j}^{r} d x^{i} d x^{j} / 2$. 't Hooft symbols are real and antisymmetric and satisfy

$$
\begin{aligned}
\eta_{i}^{r k} \eta_{k}^{s j} & =-\delta^{r s} \delta_{i}{ }^{j}+\epsilon^{r s}{ }_{t} \eta_{i}^{t j} \\
\bar{\eta}_{i}^{r k} \bar{\eta}_{k}^{s j} & =-\delta^{r s} \delta_{i}{ }^{j}+\epsilon^{r s}{ }_{t} \bar{\eta}_{i}^{t j} \\
\eta_{i}^{r k} \bar{\eta}_{k}^{s j} & =\bar{\eta}_{i}^{s k} \eta_{k}^{r j}
\end{aligned}
$$

In terms of Pauli matrices it is easy to check that

$$
\begin{array}{lll}
\eta^{1}=i \sigma_{2} \otimes \sigma_{1} & \eta^{2}=i \sigma_{2} \otimes \sigma_{3} & \eta^{3}=1 \otimes i \sigma_{2} \\
\bar{\eta}^{1}=\sigma_{1} \otimes i \sigma_{2} & \bar{\eta}^{2}=\sigma_{3} \otimes i \sigma_{2} & \bar{\eta}^{3}=i \sigma_{2} \otimes 1
\end{array}
$$

do the job.

For singly charged strings, the magnetic rotation matrices, whose eigenvalues determine the magnetic shifts, assume the form

$$
R=(1-H)(1+H)^{-1}=\left(1-H^{+}-H^{-}\right)\left(1+H^{+}+H^{-}\right)^{-1}=R_{L} R_{R}=R_{R} R_{L}
$$

where $H^{i}{ }_{j}=G^{i k} F_{k j}$. After straightforward algebra, that heavily relies on the use of $\left[H^{+}, H^{-}\right]=0,\left(H^{+}\right)^{2}=-|h|^{2} \mathbb{1}$ and $\left(H^{-}\right)^{2}=-|\bar{h}|^{2} \mathbb{1}$, with $|v|^{2} \equiv \sum_{r} v_{r}^{2}$, one finds

$$
\begin{aligned}
& R_{L}=\frac{\left(1-|h|^{2}+|\bar{h}|^{2}\right)-2 H^{+}}{\sqrt{1+2\left(|h|^{2}+|\bar{h}|^{2}\right)+\left(|h|^{2}-|\bar{h}|^{2}\right)^{2}}} \\
& R_{R}=\frac{\left(1+|h|^{2}-|\bar{h}|^{2}\right)-2 H^{-}}{\sqrt{1+2\left(|h|^{2}+|\bar{h}|^{2}\right)+\left(|h|^{2}-|\bar{h}|^{2}\right)^{2}}} .
\end{aligned}
$$


The four bosonic string coordinates transform in the $(1 / 2,1 / 2)$ representation of $S O(4)=S U(2)_{L} \times S U(2)_{R}$, and thus the magnetic shifts of the modes of singly charged spacetime bosons, with one end on an unmagnetized stack, are determined by the two pairs

$$
\beta_{Q=1}= \pm\left(\beta_{L} \pm \beta_{R}\right)
$$

with

$$
\tan \left(2 \beta_{L}\right)=\frac{2|h|}{1-|h|^{2}+|\bar{h}|^{2}} \quad, \quad \tan \left(2 \beta_{R}\right)=\frac{2|\bar{h}|}{1+|h|^{2}-|\bar{h}|^{2}} \quad .
$$

Spacetime spinors transforming in the $(1 / 2,0)$, respectively $(0,1 / 2)$, representation have their masses shifted by $\pm \beta_{L} / \pi$, respectively $\pm \beta_{R} / \pi$. Thus the only way to preserve some susy in this (singly charged) sector is having either $\beta_{L}=0$ or $\beta_{R}=0$, which is tantamount to turning on only (anti) self-dual magnetic fields, i.e. abelian (anti)instantons on $\mathbf{T}^{4}$.

In the self-dual case, choosing as reference complex structure, e.g. $J_{i j}=\bar{\eta}_{i j}^{3}$, i.e. setting $z^{1}=x^{1}+i x^{2}$ and $z^{2}=x^{3}+i x^{4}$, shows that the magnetic two-form is a type $(1,1)$ form, i.e. $F_{(2,0)}=0$, with $F_{(1,1)} \wedge J=0$. In mathematical terms, one is dealing with a hermitean connection on a holomorphic stable bundle. The choice of complex coordinates, though particularly suitable to exposing susy and reducing the problem to a two-dimensional one, hides the beautiful hyperkähler structure coded in the ADHM construction of instantons on $\mathbf{T}^{4}$ or on its susy preserving orbifolds $\mathbf{T}^{4} / \Gamma \approx \mathbf{K} 3$.

For doubly charged strings, the situation is analogous and one gets

$$
\beta_{Q=2}= \pm 2\left(\beta_{L} \pm \beta_{R}\right)
$$

for spacetime bosons and $\beta_{Q=2}= \pm 2 \beta_{L}$ or $\beta_{Q=2}= \pm 2 \beta_{R}$ for spacetime fermions.

The situation is slightly more involved for open strings ending on branes with different magnetic fields, i.e. carrying charge $q_{a}= \pm 1$ with respect to the $U(1)_{a}$ subgroup associated to the first stack of $N_{a}$ branes and charge $q_{b}= \pm 1$ with respect to the $U(1)_{b}$ subgroup associated to the second stack of $N_{b}$ branes. Yet exploiting the close analogy between 't Hooft symbols, or rather the $4 \times 4$ matrices $\Sigma^{a}=i \eta^{a}$ and $\bar{\Sigma}^{a}=i \bar{\eta}^{a}$, and Pauli matrices $\sigma^{a}$, it takes a few minutes thinking to convince oneself that the relevant matrices

$$
R_{a b L}=R_{a L}^{q_{a}} R_{b L}^{q_{b}} \quad, \quad R_{a b R}=R_{a R}^{q_{a}} R_{b R}^{q_{b}}
$$

that determine $R_{a b}=R_{a b L} R_{a b R}=R_{a b R} R_{a b L}$ can be conveniently expressed in the form

$$
R_{L}^{q}=\cos \left(2 \beta_{L}\right)-i q \sin \left(2 \beta_{L}\right) \hat{h} \cdot \Sigma \quad, \quad R_{L}^{q}=\cos \left(2 \beta_{R}\right)-i q \sin \left(2 \beta_{R}\right) \hat{\bar{h}} \cdot \bar{\Sigma}
$$


where $\tan \left(2 \beta_{L / R}\right)$ have been defined above and a hat over a three-component vector means the unit vector in its direction. Denoting for short $\cos \left(2 \beta_{a, b}\right)$, respectively $\sin \left(2 \beta_{a, b}\right)$, by $c_{a, b}$, respectively $s_{a, b}$, and combining the two rotations yields

$$
R_{a b L}=\left(c_{a} c_{b}-q_{a} q_{b} s_{a} s_{b} \hat{h}_{a} \cdot \hat{h}_{b}\right)-i\left(q_{a} s_{a} c_{b} \hat{h}_{a}+q_{b} c_{a} s_{b} \hat{h}_{b}+q_{a} q_{b} s_{a} s_{b} \hat{h}_{a} \times \hat{h}_{b}\right) \cdot \Sigma
$$

and

$$
R_{a b R}=\left(\bar{c}_{a} \bar{c}_{b}-q_{a} q_{b} \bar{s}_{a} \bar{s}_{b} \hat{\bar{h}}_{a} \cdot \hat{\bar{h}}_{b}\right)-i\left(q_{a} \bar{s}_{a} \bar{c}_{b} \hat{\bar{h}}_{a}+q_{b} \bar{c}_{a} \bar{s}_{b} \hat{\bar{h}}_{b}+q_{a} q_{b} \bar{s}_{a} \bar{s}_{b} \hat{\bar{h}}_{a} \times \hat{\bar{h}}_{b}\right) \cdot \bar{\Sigma}
$$

The magnetic shifts of the modes of the four bosonic coordinates (two complex pairs)

$$
\beta_{a b}= \pm \beta_{a b L} \pm \beta_{a b R}
$$

are determined by

$$
\cos \left(2 \beta_{a b L}\right)=c_{a} c_{b}-q_{a} q_{b} s_{a} s_{b} \hat{h}_{a} \cdot \hat{h}_{b} \quad, \quad \cos \left(2 \beta_{a b R}\right)=\bar{c}_{a} \bar{c}_{b}-q_{a} q_{b} \bar{s}_{a} \bar{s}_{b} \hat{\bar{h}}_{a} \cdot \hat{\bar{h}}_{b} \quad .
$$

Equivalent but less explicit formulae have been found in 15 .

Generically, there are no unmagnetized directions. Indeed $\beta_{a b} \neq 0$ unless $h_{a}= \pm \bar{h}_{a}$, in which case one is effectively restricting the fluxes to a threedimensional torus and considering the diagonal $S O(3)$ subgroup. Strings connecting branes of different kinds may however appear with non-trivial multiplicities associated to the presence of fermionic zero-modes counted by the index of the Dirac operator coupled with charge $q_{a}$ to the magnetic field $F_{a}$ on the first stack of $N_{a}$ branes and with charge $q_{b}$ to the magnetic field $F_{b}$ on the second stack of $N_{b}$ branes. The index vanishes in odd dimension. In four dimensions it is given by the second Chern number $c_{2}\left(q_{a} \mathcal{F}_{a}+q_{b} \mathcal{F}_{b}\right)$ of the abelian gauge bundle

$$
\begin{aligned}
I_{a b} & =-\frac{1}{2(2 \pi)^{2}} \int_{\Sigma^{4}} \operatorname{Tr}_{\left(N_{a}, N_{b}\right)}\left[\left(q_{a} \mathcal{F}_{a}+q_{b} \mathcal{F}_{b}\right) \wedge\left(q_{a} \mathcal{F}_{a}+q_{b} \mathcal{F}_{b}\right)\right] \\
& =\frac{W_{a} W_{b}}{(2 \pi)^{2}}\left[-\left|q_{a} h_{a}+q_{b} h_{b}\right|^{2}+\left|q_{a} \bar{h}_{a}+q_{b} \bar{h}_{b}\right|^{2}\right]
\end{aligned}
$$

where $\mathcal{F}_{a / b}$ are the world-volume magnetic fields and $W_{a / b}=\operatorname{det}\left(W_{a / b}\right)$ take care of the oriented brane wrappings so that $N_{a / b}=\hat{N}_{a / b}\left|W_{a / b}\right|$. For "parallel" magnetic fields in factorized rectangular two-tori $\mathbf{T}^{4}=\mathbf{T}_{(1)}^{2} \times \mathbf{T}_{(2)}^{2}, W_{a / b}=$ $\prod_{i=1}^{2} n_{a / b}^{(i)}$ and (31) reduces to the simpler expression

$$
I_{a b}=\frac{W_{a} W_{b}}{(2 \pi)^{2}} \prod_{i=1}^{2}\left(q_{a} h_{a}^{(i)}+q_{b} h_{b}^{(i)}\right)=\prod_{i=1}^{2}\left(q_{a} m_{a}^{(i)} n_{b}^{(i)}+q_{b} m_{b}^{(i)} n_{a}^{(i)}\right)
$$


upon imposing Dirac quantization condition on each subtorus and for each stack of branes. In summary, there are $I_{a b}$ strings in the representation $\left(\hat{N}_{a}, \hat{N}_{b}{ }^{*}\right)$ or $\left(\hat{N}_{a}, \hat{N}_{b}^{*}\right)$, present only in the unoriented case, of the ChanPaton group commuting with the magnetic $U(1)$ 's. The analysis of the doubly charged strings, present only in the unoriented case, is similar. In order to correctly determine the multiplicities one has to properly take into account the action of $\Omega$ that (anti) symmetrizes the Chan-Paton indices.

Although we have all the necessary ingredients to write down the oneloop partition function for a magnetized four-torus, we refrain from doing so in order to avoid cumbersome formulae that can be conveniently subsumed by the following observations. As in the two-dimensional case, one has to distinguish several sectors.

Neutral strings with $q_{a}=q_{b}=0$, starting and ending on unmagnetized branes, suffer neither magnetic shifts of their oscillator modes nor rescalings of their KK momenta.

Dipole strings with $q_{a}=-q_{b}= \pm 1$, starting and ending on the same stack of magnetized branes, suffer no magnetic shifts but carry rescaled KK momenta.

Singly charged strings $q_{a}= \pm 1$ and $q_{b}=0$ or vice versa, starting on a stack of magnetized branes and ending on a stack of unmagnetized branes, suffer magnetic shifts given by (23) and, generically, carry no KK momenta but rather multiplicities determined by the index theorem (31) to be $c_{2}\left(\mathcal{F}_{a}\right)$.

Doubly charged strings with $q_{a}=q_{\tilde{a}}= \pm 1$, starting on magnetized branes and ending on their images under $\Omega$, suffer double magnetic shifts and, generically, carry no KK momenta but rather multiplicities determined by the index theorem to be $c_{2}\left( \pm 2 \mathcal{F}_{a}\right)= \pm 4 c_{2}\left(\mathcal{F}_{a}\right)$, up to (anti)symmetrization imposed by $\Omega$.

Dy-charged strings with $q_{a}= \pm 1$ and $q_{b}= \pm 1$, starting on a stack of magnetized branes and ending on a different stack of magnetized branes or their images under $\Omega$, suffer magnetic shifts determined by the trigonometric formula (30), generically carry no KK momenta but rather discrete multiplicities determined by the index theorem, i.e. $c_{2}\left(q_{a} \mathcal{F}_{a}+q_{b} \mathcal{F}_{b}\right)$ up to identifications resulting from $\Omega$.

So far we have tacitly assumed a square torus and vanishing NS-NS antisymmetric tensor background. For an arbitrary torus one needs to replace the coordinate differentials $d x^{i}$ with the tetrad $E^{\alpha}=E_{i}^{\alpha} d x^{i}$ so that $G_{i j}=$ $\delta_{\alpha \beta} E_{i}^{\alpha} E_{j}^{\beta}$. This may not be enough in the case of non-trivial wrapping whereby $W_{(a) \alpha}^{i} \neq \delta_{\alpha}^{i}$ for some $a$. In the absence of magnetic field the integers $W_{a}=$ $\operatorname{det}\left(W_{\alpha(a)}^{i}\right)$ simply rescale the tension of the stack of branes $\tau_{a}=\tau N_{a}\left|W_{a}\right|$ and 
their R-R charge density $\rho_{a}=\tau N_{a} W_{a}$, so that the stacks preserve the same 16 supercharges only when $W_{a} W_{b}>0$ for all $a$ and $b$. Moreover in type I theories this fraction coincides with the one preserved by standard $\Omega$-planes for a given choice of sign, let us say $W_{a}>0$. For vanishing NS-NS antisymmetric tensor background, R-R tadpole cancellation requires $2 \sum_{a} N_{a} W_{a}=32$, after including image branes. The situation becomes more interesting when some magnetic fields are turned on. The subtle point is that the magnetic field that satisfies Dirac quantization condition is the worldvolume magnetic field $\mathcal{F}_{\alpha \beta}^{a}$ rather than $F_{i j}$, that need not have integer 'periods', i.e. first Chern numbers $c_{i j}$. Rather $c_{\alpha \beta}=W_{\alpha}{ }^{i} W_{\beta}{ }^{j} c_{i j}$ are integrally quantized, except possibly for a half-integer shift related to the presence of (the pull-back of) a quantized NSNS antisymmetric tensor background $\mathcal{B}_{\alpha \beta}=W_{\alpha}{ }^{i} W_{\beta}{ }^{j} B_{i j}$. As observed by [21], this may not be sufficient. In general, one has to require integrality of all Chern numbers, which are T-dual to all possible (sub)brane charges. In practise, one has to solve the inverse problem, i.e. given rational numbers $c_{i j}^{1}, c_{i j, k l}^{2}$, etc, one should find an integer matrix $W_{\alpha}^{i}$ such that $c_{\alpha \beta}^{1}, c_{\alpha \beta, \gamma \delta}^{2}$, etc are (half) integer compatibly with $\mathrm{R}-\mathrm{R}$ tadpole conditions. This may fail with purely abelian fields. In some cases [21, adding a non-abelian part might help imposing integrality but would require abandoning the exact worldsheet analysis of the present paper.

We are now ready to discuss the potential for the closed string moduli and address the issue of their stabilization. The potential is generated by the Born-Infeld lagrangian 40]

$$
\sqrt{\operatorname{det}\left(\mathcal{G}_{\alpha \beta}+\mathcal{F}_{\alpha \beta}\right)}=\sqrt{\operatorname{det}\left[W_{\alpha}{ }^{i} W_{\beta}{ }^{j}\left(G_{i j}+F_{i j}\right)\right]}=\left|\operatorname{det}\left(W_{\alpha}^{i}\right)\right| \sqrt{\operatorname{det}\left(G_{i j}+F_{i j}\right)}
$$

and the WZ coupling to the R-R fields

$$
\begin{aligned}
& \int_{\Sigma^{d}} \sum_{k} \mathcal{C}_{k+1} \operatorname{Tr}\left(e^{\mathcal{F}}\right)=\sum_{a} W_{a}\left[C_{10-d} \int_{\mathbf{T}^{d}} \omega_{d} N_{a}+\sum_{f} C_{8-d+2}^{f} \int_{\mathbf{T}^{d}} \chi_{d-2}^{r} \wedge F_{a}\right. \\
& \left.+\sum_{p} C_{6-d+2}^{q} \int_{\mathbf{T}^{d}} \psi^{q} \wedge F_{a} \wedge F_{a}+\sum_{u} C_{4-d+2}^{u} \int_{\mathbf{T}^{d}} \varphi^{u} \wedge F_{a} \wedge F_{a} \wedge F_{a}\right]
\end{aligned}
$$

Terms with an odd number of magnetic fields disappear upon $\Omega$-projection. Notice that once $\operatorname{det}\left(W_{\alpha}^{i}\right)$ is factored out it is the non-integrally (but rationally) quantized $F_{a}$ that appears. The Born-Infeld term [40] yields

$$
\sqrt{\operatorname{det}\left(\mathcal{G}_{i j}+\mathcal{F}_{i j}\right)}=\sqrt{\operatorname{det}\left(\mathcal{G}_{i j}\right)} \sqrt{\operatorname{det}(\mathbb{1}+\mathcal{H})}
$$


where $\mathcal{H}^{i}{ }_{j}=\mathcal{G}^{i k} \mathcal{F}_{k j}$. In turn

$$
\operatorname{det}(\mathbb{1}+\mathcal{H})=\exp \left[\operatorname{tr}(\log (\mathbb{1}+\mathcal{H})]=\exp \left[\sum_{k=0}^{[d / 2]} \frac{1}{2 k} \operatorname{tr}\left(\mathcal{H}^{2 k}\right)\right]\right.
$$

where $\operatorname{tr}(.$.$) denote a trace over target space vector indices, not to be confused$ with $\operatorname{Tr}(.$.$) denoting a trace over Chan-Paton indices. Indeed it is easy to$ show that $\operatorname{tr}\left(\mathcal{H}^{2 k+1}\right)=0$. Similarly the restriction to $k<[d / 2]$ can be reached via group theory considerations as well as inspection of the initial determinant that can expose terms with at most $\mathcal{F}^{d}$.

More explicitly, for $d=2,3$

$$
\frac{\operatorname{det}(\mathcal{G}+\mathcal{F})}{\operatorname{det}(\mathcal{G})}=\left[1-\frac{1}{2} \operatorname{tr}\left(\mathcal{H}^{2}\right)\right]=\left(1+h^{2}\right)
$$

where $h$ is the skew eigenvalue of $\mathcal{H}$.

Similarly, for $d=4,5$, one has

$$
\frac{\operatorname{det}(\mathcal{G}+\mathcal{F})}{\operatorname{det}(\mathcal{G})}=\left[1-\frac{1}{2} \operatorname{tr}\left(\mathcal{H}^{2}\right)-\frac{1}{4} \operatorname{tr}\left(\mathcal{H}^{4}\right)+\frac{1}{8}\left(\operatorname{tr}\left(\mathcal{H}^{2}\right)\right)^{2}\right]=\left(1+h_{1}^{2}\right)\left(1+h_{2}^{2}\right)
$$

where $h_{i}$ with $i=1,2$ are the two skew eigenvalues of $\mathcal{H}$.

Finally, for $d=6,7$, one finds

$$
\begin{aligned}
& \frac{\operatorname{det}(\mathcal{G}+\mathcal{F})}{\operatorname{det}(\mathcal{G})}=\left[1-\frac{1}{2} \operatorname{tr}\left(\mathcal{H}^{2}\right)-\frac{1}{4} \operatorname{tr}\left(\mathcal{H}^{4}\right)+\frac{1}{8}\left(\operatorname{tr}\left(\mathcal{H}^{2}\right)\right)^{2}-\right. \\
& \left.\quad-\frac{1}{6} \operatorname{tr}\left(\mathcal{H}^{6}\right)+\frac{1}{8} \operatorname{tr}\left(\mathcal{H}^{2}\right) \operatorname{tr}\left(\mathcal{H}^{4}\right)-\frac{1}{48}\left(\operatorname{tr}\left(\mathcal{H}^{2}\right)\right)^{3}\right]=\prod_{i=1}^{3}\left(1+h_{i}^{2}\right)
\end{aligned}
$$

where $h_{i}$ with $i=1,2,3$ are the skew eigenvalues of $\mathcal{H}$.

Let us now specialize to $d=4$, where one can exploit the hyperkähler structure of $\mathbf{T}^{4}$ and decompose $\mathcal{H}$ into its self-dual and anti-self-dual parts $\mathcal{H}=\mathcal{H}_{+}+\mathcal{H}_{-}$. Then one can rewrite (38) as

$$
\begin{aligned}
& 1-\frac{1}{2} \operatorname{tr}\left(\mathcal{H}^{2}\right)-\frac{1}{4} \operatorname{tr}\left(\mathcal{H}^{4}\right)+\frac{1}{8}\left(\operatorname{tr}\left(\mathcal{H}^{2}\right)\right)^{2}= \\
& 1-\frac{1}{2}\left[\operatorname{tr}\left(\mathcal{H}_{+}^{2}\right)+\operatorname{tr}\left(\mathcal{H}_{-}^{2}\right)\right]+\frac{1}{16}\left[\operatorname{tr}\left(\mathcal{H}_{+}^{2}\right)-\operatorname{tr}\left(\mathcal{H}_{-}^{2}\right)\right]^{2} .
\end{aligned}
$$

Combining with the volume factor, the Born-Infeld lagrangian of each stack of branes can be elegantly written in the form

$$
\begin{aligned}
& \sqrt{\operatorname{det}(\mathcal{G})}\left[\left(1 \mp \frac{1}{2}\left[\operatorname{tr}\left(\mathcal{H}_{+}^{2}\right)-\operatorname{tr}\left(\mathcal{H}_{-}^{2}\right)\right]\right)^{2}+\operatorname{tr}\left(\mathcal{H}_{\mp}^{2}\right)\right]^{1 / 2}= \\
& |W|\left[\left(\frac{1}{2} \int J \wedge J-\frac{1}{2} \int F \wedge F\right)^{2}+\left(\int J \wedge F\right)^{2}+\left|\int \Omega \wedge F\right|^{2}\right]^{1 / 2}
\end{aligned}
$$


where $\Omega=J^{1}+i J^{2}=J^{+}$is the complex $(2,0)$-form and $J=J^{3}$ is the Kähler form in the reference complex structure. Our normalizations are such that $\Omega \wedge \bar{\Omega}=2 J \wedge J$. At the linear level the susy preserving BPS conditions require that either $\mathcal{H}_{+}=0$ or $\mathcal{H}_{-}=0$ for all magnetic $U(1)$ 's. In the latter case, $\mathcal{H}_{-}=0$, the last three terms drop out and one ends up with

$$
\sqrt{\operatorname{det}(\mathcal{G})}\left|1-\frac{1}{2} \operatorname{tr}\left(\mathcal{H}_{+}^{2}\right)\right|=\sqrt{\operatorname{det}(\mathcal{G})}\left(1-\frac{1}{2} \operatorname{tr}\left(\mathcal{H}_{+}^{2}\right)\right) .
$$

Indeed, both terms are positive definite since $\mathcal{H}_{+}$being real and antisymmetric is anti-hermitean. The same is true in the former case, $\mathcal{H}_{+}=0$, after reversing the orientation of $\mathbf{T}^{4}$.

Contrary to the 2- and 3-dimensional cases, the 1/2-loop potential for the NS-NS moduli induced by the Born-Infeld term combined with the contribution of $\Omega$-planes depends a priori on all the moduli. Rescaling the metric according to $\widehat{G}=G / \operatorname{det}(G)^{1 / 4}$, it can be cast into the form

$$
V\left(\phi, \omega, \hat{G} ; \mathcal{F}_{a}\right)=\tau e^{-\phi}\left(2 \sum_{a} N_{a} \sqrt{\left|W_{a}\right|^{2} \omega^{2}+\left|W_{a}\right| \omega c_{1}^{2}\left(\mathcal{F}_{a}\right)+c_{2}^{2}\left(\mathcal{F}_{a}\right)} \mp 32|\omega|\right)
$$

where $\omega=\sqrt{\operatorname{det}(G)}$ parametrizes the overall volume of $\mathbf{T}^{4}$. Depending on whether $c_{1}^{2}\left(\mathcal{F}_{a}\right)$ is larger or smaller than $2\left|c_{2}\left(\mathcal{F}_{a}\right)\right|$ and on the sign of the $\Omega$-plane tension one gets different behaviours for $\omega$. For supersymmetric configurations $c_{1}^{2}\left(\mathcal{F}_{a}\right)= \pm 2 c_{2}\left(\mathcal{F}_{a}\right)$ (the same sign for all $a$ ) and one simply gets

$$
V\left(\phi, \omega, \hat{G} ; F_{a}\right)=\tau e^{-\phi}\left(2 \sum_{a} N_{a}\left(\left|W_{a}\right||\omega|+\left|c_{2}\left(\mathcal{F}_{a}\right)\right|\right) \mp 32|\omega|\right)
$$

this should be supplemented with the R-R tadpole conditions $2 \sum_{a} N_{a} W_{a}=$ \pm 32 (for $C_{10}$ ) and $2 \sum_{a} N_{a} W_{a} c_{2}\left(F_{a}\right)=0$ (for $C_{6}$ ) that cannot be satisfied for non-trivial magnetic fields unless one introduces lower dimensional $\Omega$-planes.

For non supersymmetric configurations, R-R tadpoles can be satisfied and in principle all the closed string moduli, including $\omega$ yet excluding $\phi$, can be stabilized in the presence of enough stacks of branes with oblique magnetic fluxes. One cannot prevent however the presence of open string tachyons that destabilize the vacuum anyway.

We would like to conclude this section with an amusing observation on non-linear self-duality [22, 33. As discussed above, barring the overall volume factor, the Born-Infeld action in $d=4$ can be compactly written as

$$
\sqrt{\operatorname{det}(1+\mathcal{H})}=\sqrt{\left(1+h_{1}^{2}\right)\left(1+h_{2}^{2}\right)}=\sqrt{\left(1 \pm h_{1} h_{2}\right)^{2}+\left(h_{1} \mp h_{2}\right)^{2}}
$$


where $h_{i}$ with $i=1,2$ are the two skew eigenvalues of $H$. "Standard" (anti) self-dual and thus supersymmetric configurations correspond to $h_{1} \mp h_{2}=0$ and yield

$$
\sqrt{\operatorname{det}(1+\mathcal{H})}=\left|1 \pm h_{1} h_{2}\right|=1+|h|^{2}
$$

that, combined with the R-R coupling, disclose the presence of BPS D5- or $\overline{\mathrm{D}} 5$-branes inside the magnetic D9-branes [23. The resulting 1/4 BPS bound state is at threshold since the positive tensions simply add. In order to 'dispose of' the square root in (44) and find other bound states at threshold one can however consider the case $1 \pm h_{1} h_{2}=0$, i.e. $h_{1}= \pm 1 / h_{2}$ that does not lead to standard BPS branes but corresponds to a non (manifestly) BPS state which is T-dual to a BPS configuration of D5-branes bound to pairs of D9- and D9branes. It is natural to ask to what extent the initial configuration satisfying the non-linear (anti) self-duality condition is non-BPS. It is not unconceivable that BPS-ness can be regained, even before perfoming any T-duality, with respect to local supersymmetries associated to gravitini in the closed string spectrum that become massless at the non-linear (anti) self-dual point.

\section{Five-dimensional magnetized torus}

A constant magnetic field $F$ in five dimensions has 10 independent components. Four of them may be taken to be associated to the independent components of the unit vector $\vec{u}$ along the "unmagnetized" direction associated to the unit eigenvalue of the rotation matrix $R(F)$. The other six determine the magnetic field in the four transverse generically "magnetized" directions. By means of this splitting one can easily determine the magnetic shifts for singly and doubly charged strings and the rescaling of the momenta for dipole strings. The study of dy-charged strings, although straightforward in principle, is quite laborious in practice since one has to diagonalize a generic $5 \times 5$ orthogonal matrix, something that leads to very cumbersome formulae. Yet, the strategy is first to identify the "unmagnetized" direction by solving $R_{a b} \vec{u}_{a b}=\vec{u}_{a b}$, then decompose the reduced $4 \times 4$ orthogonal matrix according to $R_{a b}^{\perp}=R_{a b}^{\perp L} R_{a b}^{\perp R}$ and finally exploit the properties of 't Hooft symbols in order to determine the eigenvalues and the resulting magnetic shifts.

The condition for supersymmetry in $D=5$ are as stringent as in $D=6$. In concrete terms the magnetic rotation matrices should all belong to the same $S U(2)=S p(2)$ subgroup of $S O(5)=S p(4)$. In particular this means that they should all have the same unmagnetized direction $\vec{u}$, which may or may 
not coincide with one of the generators of the fundamental cell. The holonomy of the $S O(4)$ stability group should further reduce to $S U(2)$.

Since nothing essentially new can be learned from this case we do not go into any further detail.

\section{Six-dimensional magnetized torus}

Six dimensional magnetized tori represent a genuinely new and phenomenologically interesting case. In general, a magnetic field has 15 independent components, corresponding to the constant and thus closed two-forms. For obvious (supersymmetry) purposes it is convenient to choose complex coordinates and set

$$
F^{a}=F_{(2,0)}^{a}+F_{(1,1)}^{a}+F_{(0,2)}^{a}
$$

where $F_{(2,0)}^{a}, F_{(1,1)}^{a}$ and $F_{(0,2)}^{a}$ have 6,9 and 6 independent components respectively. Notice that $F_{(0,2)}^{a}=F_{(2,0)}^{a *}, F_{(1,1)}^{a}=F_{(1,1)}^{a *}$ for a real gauge field, i.e. a hermitean connection.

In order to preserve some supersymmetry [22, 20, 33, the complex structure of $\mathbf{T}^{6}$ must be chosen so that for all $a$

$$
F_{(0,2)}^{a}=0
$$

and the Kähler form $J=i G_{i j} d z^{i} \wedge d z^{\bar{j}}$ must satisfy

$$
\frac{1}{2 !} J \wedge J \wedge F_{(1,1)}^{a}=\frac{1}{3 !} F_{(1,1)}^{a} \wedge F_{(1,1)}^{a} \wedge F_{(1,1)}^{a},
$$

i.e. the magnetic bundle must be holomorphic and stable wrt to $J$. In turn this implies a reduction of the holonomy group generated by the magnetic rotation matrices $R_{a}$ from $S O(6)=S U(4)$ to $S U(3)$ for $\mathcal{N}=1$ supersymmetry or $S U(2)$ for $\mathcal{N}=2$ supersymmetry. $\mathcal{N}=4$ supersymmetry is enjoyed by the neutral open strings, for which $R_{0}=\mathbb{1}$.

In the supersymmetric case, the $6 \times 6$ rotation matrices are direct sums of $3 \times 3$ blocks

$$
R=\left(\begin{array}{cc}
U_{\mathbf{3}} & 0 \\
0 & U_{\mathbf{3}^{*}}
\end{array}\right)
$$

since $\mathbf{6} \rightarrow \mathbf{3}+\mathbf{3}^{*}$ under $S U(4) \rightarrow U(3)$. More explicitly

$$
U_{\mathbf{3} j}^{i}=\left(\delta^{i}{ }_{k}-i H_{k}^{i}\right)\left(\delta^{k}{ }_{j}+i H^{k}{ }_{j}\right)^{-1}
$$

where $H^{i}{ }_{j}=G^{i \bar{k}} F_{\bar{k} j}$ and similarly for $U_{\mathbf{3}^{*}}$. 
The stability condition is not simply $H^{i}{ }_{i}=0$, as in $d=4$ where it arose from $J \wedge F=0$, but rather the condition for $S U(3)$ holonomy: $\operatorname{det}\left(U_{\mathbf{3}}\right)=1$. In other words $\operatorname{det}(1+i H)=\operatorname{det}(1-i H)$ that implies $\sum_{k}(-)^{k} \operatorname{tr}\left(H^{2 k+1}\right) /(2 k+1)=$ 0 . Since in general $\operatorname{tr}_{\mathbf{3}}(H) \neq 0$, one can expand $H_{3}$ in the basis of generators of $U(3)$

$$
H=h_{0} \mathbb{1}+h_{r} \lambda^{r}
$$

where we choose the eight $3 \times 3$ Gell-Mann matrices $\lambda^{r}$ so as to be hermitean and satisfy

$$
\operatorname{tr}\left(\lambda^{r} \lambda^{s}\right)=3 \delta^{r s} \quad \lambda^{r} \lambda^{s}=\delta^{r s} \mathbb{1}+\left(i f^{r s t}+d^{r s t}\right) \lambda_{t},
$$

where the $S U(3)$ structure constants $f^{r s t}$ are totally antisymmetric and the anomaly coefficients $d^{r s t}$ are totally symmetric.

In order to determine the eigenvalues $\rho_{k}=\exp \left(2 i \beta_{k}\right)$ of $U$ in terms of the 9 components $h_{0}$ and $h_{r}$ of $H$ it is convenient to diagonalize $\hat{H}=h_{r} \lambda^{r}=h \cdot \lambda$ so that

$$
\rho_{k}=\frac{1-i h_{0}-i \mu_{k}}{1+i h_{0}+i \mu_{k}}
$$

Using $\operatorname{tr}(\hat{H})=0, \operatorname{tr}\left(\hat{H}^{2}\right)=3 \delta^{r s} h_{r} h_{s} \equiv 3|h|^{2}$ and $\operatorname{tr}\left(\hat{H}^{3}\right)=3 d^{r s t} h_{r} h_{s} h_{t} \equiv$ $3 d h h h$, the secular equation for $\hat{H}$ boils down to a cubic in canonical form

$$
\mu^{3}-\frac{3}{2}|h|^{2} \mu-d h h h=0 \quad,
$$

whose solutions are given by Cardano - Tartaglia formula

$$
\begin{aligned}
\mu_{k} & =\frac{e^{2 k \pi i / 3}}{2^{1 / 3}}\left[d h h h+i \sqrt{\frac{1}{2}|h|^{6}-(d h h h)^{2}}\right]^{1 / 3} \\
& +\frac{e^{-2 k \pi i / 3}}{2^{1 / 3}}\left[d h h h-i \sqrt{\frac{1}{2}|h|^{6}-(d h h h)^{2}}\right]^{1 / 3},
\end{aligned}
$$

where $|h|^{6} \geq 2(d h h h)^{2}$ has been used. Supersymmetry translates into the condition

$$
h_{0}^{3}+h_{0}^{2} \sum_{k} \mu_{k}+h_{0}\left(\sum_{i<j} \mu_{i} \mu_{j}-3\right)+\prod_{k} \mu_{k}-\sum_{k} \mu_{k}=0 \quad .
$$

We now consider the more laborious case of strings ending on different stacks of branes, whose magnetic shifts are determined by the eigenvalues of 
the $S U(3)$ matrix $U_{a b}=U_{a}^{q_{a}} U_{b}^{q_{b}}$. Setting for definiteness $q_{a}=1$ and $q_{b}=-1$ and factoring out an overall $U(1)$ phase gives

$$
\begin{aligned}
U_{a b} & =\frac{\left(1-i h_{a}^{0}\right)\left(1+i h_{b}^{0}\right)}{\left(1+i h_{a}^{0}\right)\left(1-i h_{b}^{0}\right)}\left(1-i \hat{h}_{a} \cdot \lambda\right)\left(1+i \hat{h}_{a}^{*} \cdot \lambda\right)^{-1}\left(1-i \hat{h}_{b}^{*} \cdot \lambda\right)\left(1+i \hat{h}_{b} \cdot \lambda\right)^{-1} \\
& =\frac{\left(1-i h_{a}^{0}\right)\left(1+i h_{b}^{0}\right)}{\left(1+i h_{a}^{0}\right)\left(1-i h_{b}^{0}\right)} \hat{U}_{a b}
\end{aligned}
$$

where $\hat{h}_{a / b}=h_{a / b} /\left(1-i h_{a / b}^{0}\right)$ and $*$ denotes complex conjugation. Perusing (52) yields

$$
\hat{U}_{a b}=M_{a}^{-1} \frac{1-\hat{h}_{a} \cdot \hat{h}_{b}}{1-\hat{h}_{a}^{*} \cdot \hat{h}_{b}^{*}}\left(1-i \hat{h}_{a b} \cdot \lambda\right)\left(1+i \hat{h}_{a b}^{*} \cdot \lambda\right)^{-1} M_{a}
$$

where $M_{a}=1+i \hat{h}_{a}^{*} \cdot \lambda$ and

$$
\hat{h}_{a b}=\frac{\hat{h}_{a}+\hat{h}_{b}+f \hat{h}_{a} \hat{h}_{b}-i d \hat{h}_{a} \hat{h}_{b}}{1-\hat{h}_{a} \cdot \hat{h}_{b}}
$$

so that

$$
\hat{\mu}_{a b, k}=\frac{1-\hat{h}_{a} \cdot \hat{h}_{b}}{1-\hat{h}_{a}^{*} \cdot \hat{h}_{b}^{*}} \hat{\nu}_{a b, k}
$$

where

$$
\begin{aligned}
\hat{\nu}_{a b, k} & =\frac{e^{2 k \pi i / 3}}{2^{1 / 3}}\left[d \hat{h}_{a b} \hat{h}_{a b} \hat{h}_{a b}+i \sqrt{\frac{1}{2}\left|\hat{h}_{a b}\right|^{6}-\left(d \hat{h}_{a b} \hat{h}_{a b} \hat{h}_{a b}\right)^{2}}\right]^{1 / 3} \\
& +\frac{e^{-2 k \pi i / 3}}{2^{1 / 3}}\left[d \hat{h}_{a b} \hat{h}_{a b} \hat{h}_{a b}-i \sqrt{\frac{1}{2}\left|\hat{h}_{a b}\right|^{6}-\left(d \hat{h}_{a b} \hat{h}_{a b} \hat{h}_{a b}\right)^{2}}\right]^{1 / 3}
\end{aligned}
$$

and eventually one can compute $\rho_{a b, k}=\exp \left(2 i \beta_{a b, k}\right)$ and get the magnetic shifts $\epsilon_{a b, k}=\beta_{a b, k} / \pi$, where $k=1,2,3$ labels the three orthogonal directions along the eigenevectors of $U_{a b}$. Clearly the eigenvalues of $R$ come in complex conjugate pairs $\left(\rho_{k}, \bar{\rho}_{k}\right)$. Though slightly unfamiliar, the explicit and to some extent compact expressions for the eigenvalues of $H$ and $R$ may prove very useful in extracting the spectrum and some couplings. Equivalent but less explicit formulae have been found in [15. As an illustration of our procedure, we have computed all the magnetic shifts relevant to determine the spectrum of the $\mathcal{N}=1$ supersymmetric AM model constructed by Antoniadis and Maillard [20]. The not so inspiring results are gathered in the Appendix. 
As usual multiplicities, associated to the degeneracy of the Landau levels, are counted by the relevant index theorem. In six dimensions it is given by the third Chern number $c_{2}\left(q_{a} \mathcal{F}_{a}+q_{b} \mathcal{F}_{b}\right)$ of the abelian gauge bundle

$$
\begin{aligned}
I_{a b} & =-\frac{1}{3 !(2 \pi)^{3}} \int_{\Sigma^{6}} \operatorname{Tr}_{\left(N_{a}, N_{b}\right)}\left[\left(q_{a} \mathcal{F}_{a}+q_{b} \mathcal{F}_{b}\right)^{3}\right] \\
& =-\frac{W_{a} W_{b}}{3 !(2 \pi)^{3}} \int_{\mathbf{T}^{6}} \operatorname{Tr}_{\left(N_{a}, N_{b}\right)}\left[\left(q_{a} F_{a}+q_{b} F_{b}\right)^{3}\right] .
\end{aligned}
$$

For "parallel" magnetic fields in factorized rectangular two-tori $\mathbf{T}^{6}=\mathbf{T}_{(1)}^{2} \times$ $\mathbf{T}_{(2)}^{2} \times \mathbf{T}_{(3)}^{2}, W_{a / b}=\prod_{i=1}^{3} n_{a / b}^{(i)}$ and (62) reduces to the simpler expression

$$
I_{a b}=\frac{W_{a} W_{b}}{(2 \pi)^{3}} \prod_{i=1}^{3}\left(q_{a} h_{a}^{(i)}+q_{b} h_{b}^{(i)}\right)=\prod_{i=1}^{3}\left(q_{a} m_{a}^{(i)} n_{b}^{(i)}+q_{b} m_{b}^{(i)} n_{a}^{(i)}\right)
$$

upon imposing Dirac quantization condition on each subtorus and for each stack of branes. When $I_{a b}=0$ one has no chiral asymmetry that may sometimes signal the presence of one or more unmagnetized directions. This is the case e.g. for the AM model described in the appendix. One can define a reduced index $I_{a b}^{\perp}$ in the subspace orthogonal to the fixed tori that coun their number. Clearly in such cases, dycharged strings can carry generalized KK momenta in the unmagnetized directions.

We then turn to address the issue of moduli stabilization that relies on the potential for the closed string moduli generated by the abelian and thus mutually commuting (in the Chan-Paton sense!) magnetic fluxes. The BornInfeld action, compactly expressed in terms of the skew eigenvalues of $H_{6}$ as in (39), can be rewritten as

$$
\sqrt{\prod_{i}\left(1+h_{i}^{2}\right)}=\sqrt{\left(1-h_{1} h_{2}-h_{2} h_{3}-h_{3} h_{1}\right)^{2}+\left(h_{1} h_{2} h_{3}-h_{1}-h_{2}-h_{3}\right)^{2}}
$$

up to an overall factor of $\sqrt{\operatorname{det}(\mathcal{G})}$. Expressing $h_{k}$ in terms of the available $S O(6)$ invariants, i.e. $\operatorname{tr}\left(H^{2}\right), \operatorname{tr}\left(H^{4}\right)$, and $\operatorname{tr}\left(H^{6}\right)$ or, equivalently, $\varepsilon H H H=$ $-\operatorname{tr}\left(H^{6}\right) / 6+\operatorname{tr}\left(H^{2}\right) \operatorname{tr}\left(H^{4}\right) / 8-\operatorname{tr}\left(H^{2}\right)^{3} / 48$, requires solving a complete cubic equation. For $F_{(2,0)}=0$, we have already solved the problem, since $H_{6}=$ $H_{3}+H_{3^{*}}$ and $h_{k}= \pm \mu_{k}$. In turn, $\mu_{k}$ are given in terms of the only available $U(3)$ invariants $h_{0},|h|^{2}$, and $d h h h$. Combining with the volume factor yields 22

$$
\frac{|W|}{3 !} \sqrt{\left(\int J \wedge J \wedge J-3 \int J \wedge F \wedge F\right)^{2}+\left(\int F \wedge F \wedge F-3 \int J \wedge J \wedge F\right)^{2}}
$$


In a supersymmetric configuration the last term is absent due to (48) or equivalently to the condition

$$
h_{1} h_{2} h_{3}=h_{1}+h_{2}+h_{3}
$$

which can be interpreted in geometric terms as saying that $\beta_{i}=\arctan \left(h_{i}\right)$ are the three internal angles of a triangle. As a result the effective tension, which is positive as a whole may have negative contribution on some subtori. This is exactly what is needed to cancel R-R tadpole while preserving susy and without introducing lower dimensional $\Omega$-planes [?, 20, 33]. In a sense magnetized branes behave as bound states of lower dimensional branes and anti-branes that can carry (partial) tensions and charges of different signs. The very consistency of configurations of this kind requires stabilizing the complex structure and Kähler moduli at special values depending on the magnetic fluxes. The stabilization mechanism proposed in [20] for the closed string moduli is rather simple and effective. Imposing the holomorphy condition $F_{(2,0)}^{a}=0$ for a large enough number of stacks with oblique magnetic fluxes fixes the complex structure moduli. Imposing the stability condition $F_{(1,1)}^{a} \wedge F_{(1,1)}^{a} \wedge F_{(1,1)}^{a}=3 J \wedge J \wedge F_{(1,1)}^{a}$ then fixes the Kähler moduli. This mechanism is particularly clear for the NS-NS moduli $G_{i j}$, both complex structure $G_{I J}$ and Kähler deformations $G_{I \bar{J}}$, which appear in the Born-Infeld action and for the R-R scalars $C_{I \bar{J}}$ associated to Kähler deformations which can combine with the anomalous magnetic $U(1)$ gauge fields and become massive 20, 43, 44, 45. However the R-R scalars $C_{I J}$ associated to complex structure deformations cannot directly mix with gauge fields whose internal field strenghts are of complex type $(1,1)$ at the supersymmetric point. The presence of internal magnetic fluxes, however, allows all R-R fields to mix with one another and with NS-NS fields even at zero spacetime momentum. In order to show this one has to generalize the results of [24, 25], where closed string scattering amplitudes on Dp-branes were computed, to the case of our interest, i.e. D9-branes with internal magnetic fields. Setting $\alpha^{\prime}=1$ for simplicity, the relevant amplitudes are all of the form

$$
\mathcal{A}\left(p_{1}, \xi_{1} ; p_{2}, \xi_{2}\right)=-\frac{i}{2} \kappa \tau_{p} \frac{\Gamma(s) \Gamma(t)}{\Gamma(1+t+s)} \mathcal{K}\left(p_{1}, \xi_{1} ; p_{2}, \xi_{2}\right)
$$

where $s=p_{1} D p_{1}$, respectively $t=\left(p_{1}+p_{2}\right)^{2}$, denote the kinematic invariants in the open, respectively closed, string exchange channels, while $\xi_{1}, \xi_{2}$ indicate the polarizations of the external closed string states. The boundary reflection conditions are coded in the matrix $D$. For a Dp-brane with $F=0$, one has $D_{\mu \nu}=\eta_{\mu \nu}, D_{\mu i}=D_{j \nu}=0, D_{i j}=-\delta_{i j}$. Momentum conservation along the longitudinal $p+1$ directions is understood. 
We are interested in adapting the original amplitudes to the case $F \neq 0$ in the internal directions, so that $D(F)=\mathbb{1} \oplus R(F)$ and $M(F)=\mathbb{1} \otimes \Lambda(F)$, where $\Lambda(F)$ is the spinor representation of $R(F)$. We focus on the two-point amplitude mixing an internal metric fluctuation, $\phi_{i j}=\phi_{j i}$, with an internal $\mathrm{R}-\mathrm{R}$ field, $\chi_{i j}=-\chi_{j i}$. Imposing spacetime momentum conservation only at the very end and slightly relaxing the mass-shell condition, by allowing some small non conserved internal momentum / winding, yields

$$
\mathcal{K}^{R-R / N S-N S}=\chi_{i j}^{(1)} \phi_{m n}^{(2)}\left[i \frac{s}{2 \sqrt{2}}(s+t) R^{n}{ }_{k}\left\langle\gamma^{i j} \Lambda \gamma^{k} \gamma^{m}\right\rangle+i \frac{t}{(2) \sqrt{2}} t\left\langle\gamma^{i j} \Lambda\right\rangle R^{m n}\right]
$$

where $\langle\ldots\rangle$ stands for a trace over spinor indices. The small momentum expansion of the Veneziano-like amplitude yields

$$
\frac{\Gamma(s) \Gamma(t)}{\Gamma(1+t+s)}=\frac{1}{s t}-\frac{\pi^{2}}{6}+\mathcal{O}\left(p^{4}\right)
$$

that exposes massless poles in the $s$ and $t$ channel.

A similar analysis for the scattering of massless closed strings on $\Omega$-planes gives a result of the form

$$
\mathcal{A}\left(p_{1}, \xi_{1} ; p_{2}, \xi_{2}\right)=-\frac{i}{2 t(s+t)} \kappa \tau_{\Omega} F(1-s, t ; t+1 ;-1) \mathcal{K}_{\Omega}\left(p_{1}, \xi_{1} ; p_{2}, \xi_{2}\right)
$$

where $F(1-s, t ; t+1 ;-1)=t \int_{0}^{1} d y y^{t-1}(1+y)^{s-1}$ is a hypergeometric function.

The massless $t$ channel poles of the various amplitudes correspond to closed string massless tadpoles in the NS-NS and R-R sectors. They cancel in an anomaly free supersymmetric backgrounds [46, 47, 48, 49, 50, 51, where the scalar fields extremize the potential, after summing the contributions of (magnetized) D-branes and $\Omega$-planes .

The $s$ channel poles correspond to the exchange of massless open string states that mix with the closed string states. This mixing receives no contribution from the projective plane that cannot accomodate open string channels. Indeed $F(1-s, t ; t+1 ;-1)$ is finite for $s=0$. As argued in 20] this openclosed string mixing is responsible for lifting part of the moduli, i.e. the R-R axions $\chi_{I \bar{J}}$ of type $(1,1)$ partecipating in the generalized GS mechanism of cancellation of the anomalous $U(1)$ 's that schematically reads

$$
\left\langle F^{3}\right\rangle_{1-\text { loop }}-\langle F \beta\rangle_{1 / 2-\text { loop }}\langle\beta \chi\rangle_{\text {tree }}\langle\chi F F\rangle_{1 / 2-\text { loop }}=0
$$

where $\beta_{\mu \nu K L \bar{M} \bar{N}}$ is the $\mathrm{R}-\mathrm{R}$ two-form dual (in 4-d) to the axionic $\mathrm{R}-\mathrm{R}$ scalars $\chi_{I \bar{J}}$. 
Even after subtracting the open string massless poles, there is a finite remnant that leads to R-R / NS-NS mixing of the schematic form

$$
\mathcal{L}_{m i x}=\chi_{I \bar{J}} \phi_{K \bar{L}}\left\langle\gamma^{I \bar{J}} \Lambda \gamma^{K} \gamma_{M}\right\rangle R^{M \bar{L}}+\chi_{I J} \phi_{\bar{K} \bar{L}}\left\langle\gamma^{I J} \Lambda \gamma^{\bar{K}} \gamma_{M}\right\rangle R^{M \bar{L}}
$$

These include terms responsible for the mixing of the (2,0)-type $\mathrm{R}$-R fields with the NS-NS $(2,0)$ type metric fluctuations and for their mass generation.

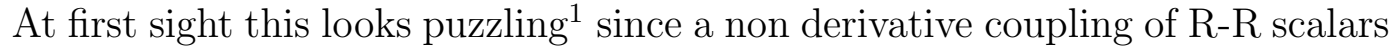
seems to violate invariance under their axionic shift symmetries. Moreover the standard form of the WZ couplings seems to prevent such mixings. However one should not forget that the R-R 6-form $C_{6}$ that appears in $S_{W Z}$ is not an elementary field but rather a composite object arising from $C_{2}$ after Poincaré duality. This in turn involves the metric as required for the mixing. Another way to see this is simply solving for $C_{6}$ and working with the modified 3 -form field strength for $C_{2}$, i.e. $H_{3}=d C_{2}+e^{\phi} \omega_{3}^{C S}$. The beating term $e^{-\phi} d C_{2} \wedge * \omega_{3}^{C S}$ in the expansion of the kinetic term for $C_{2}$ arises at the correct order (disk) and once integrated by parts can produce the desired mixing with the NS-NS metric fluctuations.

A detailed resolution of the mixing requires dealing with subtle relative normalization issues among the various amplitudes and is beyond the scope of the present investigation. In specific models, such as the AM model, one can accomplish the task with some effort and motivation. Suffice it to say that the resulting masses are highly non-trivial functions of the fluxes but, being supersymmetric, should satisfy sum rules such as $\operatorname{Str}\left(\mathcal{M}^{2}\right)=0$. In principle this could be checked by computing two-point amplitudes of closed string fermions on the disk and projective plane.

Before concluding this section let us briefly mention the role of open string moduli (Wilson lines) 28] and of additional fluxes associated to metric torsion (Scherk-Schwarz deformations [52, 53, 54]) and to internal 3-form fluxes 38. By close analogy with the heterotic string on twisted tori [36, one can easily conclude that part of the open string Wilson line moduli are eaten by the six NS-NS graviphotons $G_{\mu i}$ very much as part of the R-R axions are eaten by the 'anomalous' $U(1)$ vector bosons $A_{\mu a}$ in the open string spectrum. Adding metric torsion contributes extra mass terms for the six R-R graviphotons $B_{\mu i}$ in a perturbative setting. Indeed Scherk-Schwarz deformations of type I compactifications have been under active investigation for some time [53, 54]. In order to stabilize the dilaton and the R-R axion it seems however necessary

\footnotetext{
${ }^{1}$ M.B. would like to thank C. Angelantonj, S. Ferrara and J. F. Morales Morera for a very enlightening discussion on this topic.
} 
to turn on internal R-R 3-form fluxes ${ }^{2}$. This would spoil the nice perturbative framework of the analysis performed here. In any case it would be very important to study the conditions for moduli stabilization and unbroken susy in the presence of combined constant fluxes from the three sectors of type I strings: magnetic fields $\left(m_{i j}^{a}\right)$, NS-NS torsion à la Scherk-Schwarz $\left(\gamma_{i j}^{k}\right)$ and R-R fluxes $\left(\beta_{i j k}\right)$. We cannot help writing down the resulting non-abelian algebra satisfied by the generators of internal translations $P_{i}$, Cartan subalgebra of the Chan-Paton group $T_{a}$ and axionic shifts $W^{i}[36]$

$$
\begin{aligned}
& {\left[P_{i}, P_{j}\right]=2 i m_{i j}^{a} T_{a}+2 i \gamma_{i j}^{k} P_{k}-3 i \beta_{i j k} W^{k}} \\
& {\left[P_{i}, T^{a}\right]=-2 i m_{i j}^{a} W^{j} T_{a} \quad, \quad\left[P_{i}, W^{k}\right]=-2 i \gamma_{i j}^{k} W^{j}} \\
& {\left[W^{i}, W^{k}\right]=0 \quad, \quad\left[T_{a}, W^{k}\right]=0 \quad, \quad\left[T_{a}, T_{b}\right]=0 .}
\end{aligned}
$$

The structure constants are constrained by Jacobi identities

$$
\begin{aligned}
& \gamma_{n[i}^{l} \gamma_{k j]}^{n}=0 \quad, \quad \gamma_{k j}^{k}=0 \\
& m_{a[i j} m_{k l]}^{a}=3 \beta_{n[i j} \gamma_{k l]}^{n} \quad, \quad m_{n[k}^{a} \gamma_{i j]}^{n}=0
\end{aligned}
$$

which admit a geometric interpretation in terms of Bianchi identities for the internal p-form fields.

\section{Conclusions and perspectives}

The results presented above should have clarified several features of the dynamics of open strings coupled to oblique magnetic fields. In turn these can give a host of new possibilities for symmetry breaking, moduli stabilization and chiral asymmetry even in toroidal compactifications while preserving some residual supersymmetry. Clearly these notes were aimed a general analysis rather then the construction of specific models ${ }^{3}$ where all or some of the above effects are present. Some explicit models have been very recently discussed in [31, 32, 33., where a puzzle first raised in [55] has been successfully solved but only "parallel" magnetic field have been considered. More phenomenological aspects of oblique internal magnetic fields are under investigation [56].

Before concluding, let us briefly discuss toroidal orbifolds and models which are based on rational conformal field theories [46, 47, 50, such as Gepner

\footnotetext{
${ }^{2}$ M. B. would like to thank I. Antoniadis for a discussion on this point.

${ }^{3}$ Models with (oblique) magnetic fluxes are subject to subtle consistency conditions in order to cancel K-theory RR charges. We thank F. Marchesano for pointing this out to us.
} 
models [49, 51]. As shown in [27] for the case of the NS penta-brane, governed by the product of an $S U(2)_{k}$ WZW model and a non-compact free boson with a background charge $Q_{k}$, turning on magnetic fields corresponds to changing the phase of some boundary reflection coefficients in the transverse closed string channel. This in turn generates "twisted" representations in the direct open string channel, whose modes are shifted by an a priori rather arbitrary amount. In the case studied in [27] supersymmetry is completely broken by the introduction of magnetic fluxes but in more general cases where the chiral algebra on the worldsheet admits a spectral flow it should be possible to tune them in such a way as to preserve some supersymmetry [22, 20, 57].

As pointed out above, the non-linearity of the abelian Born-Infeld action allow for more general possibilities of non BPS brane configurations that accomodate magnetic fields stisfying a generalized non-linear (anti) self-duality condition of the type $H_{r}=1 / H_{s}$ where $r$ and $s$ label two $(1,1)$ forms. This kind of branes may offer further opportunities for phenomenologically viable type I models or their T-dual intersecting brane models [58, 59].

It is important to stress that contrary to the case of combined closed string NS-NS and R-R fluxes [37, 38, abelian magnetic fields coupled to the ends of open strings are completely under control in string perturbation theory. One can straightforwardly compute the spectrum and interactions. In the present notes we have focussed on how to derive the spectrum, in terms of magnetic shifts, multiplicities and rescalings of the internal KK momenta, but it is rather easy to generalize our analyis and extract some relevant low-energy couplings in addition to the scalar potential. Very much as in intersecting brane models which are T-dual to type I compactifications with "parallel" magnetic fluxes [60], tree-level gauge couplings are simply given by

$$
\frac{1}{g_{a}^{2}}=\left|W_{a}\right| e^{-\phi} \sqrt{\operatorname{det}\left(G+F_{a}\right)}
$$

that further simplify at supersymmetric extrema. Notice the role of the wrapping factors $\left|W_{a}\right|=\operatorname{det}\left(W_{a}\right)_{\alpha}^{i}$ in the relative rescaling of the gauge couplings wrt to one another. Due to R-R tadpole cancellation $\left|W_{a}\right|$ are also important in reducing the rank of the gauge group. The one-loop running can be determined turning on (small) magnetic fields along the non-compact spacetime directions as in 60. The role of the $B$ field has been investigated by [61]

Another important aspect that has been investigated in [20] is the possibility of accomodating some large extra dimensions 62. We hope to get back to this point in [56] together with a detailed analysis of Yukawa couplings for models with oblique internal magnetic fields extending the analysis of the 
"parallel" case in [63, 64].

\section{Acknowledgements}

We would like to thank P. Anastasopoulos, C. Angelantonj, I. Antoniadis, S. Ferrara, F. Fucito, M. Larosa, A. Lionetto, J. F. Morales Morera, M. Nicolosi, G. Pradisi, M. Prisco, R. Russo, A. Sagnotti, and Ya. Stanev for useful discussions. Our special thanks go to C. Angelantonj, G. Pradisi, A. Sagnotti for valuable comments on the manuscript. M. B. would like to thank the CERN Theory Division for its kind hospitality while this work was being completed. This work was supported in part by INFN, by the MIURCOFIN contract 2003-023852, by the EU contracts MRTN-CT-2004-503369 and MRTN-CT-2004-512194, by the INTAS contract 03-516346 and by the NATO grant PST.CLG.978785. 


\section{Appendix: the AM model}

In this appendix we display the rotation matrices $R_{a}$ and list the eigenvalues of $R_{a}^{q_{a}} R_{b}^{q_{b}}$ for the AM toroidal model constructed by Antoniadis and Maillard [20]. It consists of 9 stacks of magnetized D9-branes and one stack of unmagnetized ones. An explicit realization of moduli stabilization through "tangled" $U(1)$ fluxes is shown. In the following table, we list the number of branes and the numerical value of the internal magnetic fields at the stable supersymmetric point.

\begin{tabular}{|c|c|c|}
\hline Stacks & nagnetic and wrapping numbers & magnetic fields \\
\hline$N_{1}=1$ & $\begin{array}{l}\left(m_{x_{1} y_{2}}, n_{x_{1} y_{2}}\right)=(1,-1) \\
\left(m_{x_{2} y_{1}}, n_{x_{2} y_{1}}\right)=(1,-1) \\
\left(m_{x_{3} y_{3}}, n_{x_{3} y_{3}}\right)=(0,-1)\end{array}$ & $\begin{array}{l}H_{x_{1} y_{2}}=-\sqrt{2} \\
H_{x_{2} y_{1}}=-\sqrt{2} \\
H_{x_{3} y_{3}}=0\end{array}$ \\
\hline$N_{2}=1$ & $\begin{array}{l}\left(m_{x_{1} y_{3}}, n_{x_{1} y_{3}}\right)=(1,-1) \\
\left(m_{x_{3} y_{1}}, n_{x_{3} y_{1}}\right)=(1,-1) \\
\left(m_{x_{2} y_{2}}, n_{x_{2} y_{2}}\right)=(0,-1)\end{array}$ & $\begin{array}{l}H_{x_{1} y_{3}}=-2 \\
H_{x_{3} y_{1}}=-2 \\
H_{x_{2} y_{2}}=0\end{array}$ \\
\hline$N_{3}=1$ & $\begin{array}{l}\left(m_{x_{1} x_{2}}, n_{x_{1} x_{2}}\right)=(1,-1) \\
\left(m_{y_{1} y_{2}}, n_{y_{1} y_{2}}\right)=(1,-1) \\
\left(m_{x_{3} y_{3}}, n_{x_{3} y_{3}}\right)=(0,-1)\end{array}$ & $\begin{array}{l}H_{x_{1} x_{2}}=-\sqrt{2} \\
H_{y_{1} y_{2}}=-\sqrt{2} \\
H_{x_{3} y_{3}}=0\end{array}$ \\
\hline$N_{4}=2$ & $\begin{array}{l}\left(m_{x_{1} y_{1}}, n_{x_{1} y_{1}}\right)=(0,-1) \\
\left(m_{x_{2} x_{3}}, n_{x_{2} x_{3}}\right)=(1,-1) \\
\left(m_{y_{2} y_{3}}, n_{y_{2} y_{3}}\right)=(1,-1)\end{array}$ & $\begin{array}{l}H_{x_{1} y_{1}}=0 \\
H_{x_{2} x_{3}}=-1 \\
H_{y_{2} y_{3}}=-1\end{array}$ \\
\hline$N_{5}=1$ & $\begin{array}{l}\left(m_{x_{1} x_{3}}, n_{x_{1} x_{3}}\right)=(1,-1) \\
\left(m_{x_{2} y_{2}}, n_{x_{2} y_{2}}\right)=(0,-1) \\
\left(m_{y_{1} y_{3}}, n_{y_{1} y_{3}}\right)=(1,-1)\end{array}$ & $\begin{array}{l}H_{x_{1} x_{3}}=-2 \\
H_{x_{2} y_{2}}=0 \\
H_{y_{1} y_{3}}=-2\end{array}$ \\
\hline$N_{6}=2$ & $\begin{array}{l}\left(m_{x_{1} y_{1}}, n_{x_{1} y_{1}}\right)=(0,-1) \\
\left(m_{x_{2} y_{3}}, n_{x_{2} y_{3}}\right)=(1,-1) \\
\left(m_{x_{3} y_{2}}, n_{x_{3} y_{2}}\right)=(1,-1)\end{array}$ & $\begin{array}{l}H_{x_{1} y_{1}}=0 \\
H_{x_{2} y_{3}}=-1 \\
H_{x_{3} y_{2}}=-1\end{array}$ \\
\hline$N_{7}=2$ & $\begin{array}{c}\left(m_{x_{1} y_{1}}, n_{x_{1} y_{1}}\right)=(-2,1) \\
\left(m_{x_{2} y_{2}}, n_{x_{2} y_{2}}\right)=(0,1) \\
\left(m_{x_{3} y_{3}}, n_{x_{3} y_{3}}\right)=(1,1)\end{array}$ & $\begin{aligned} H_{x_{1} y_{1}} & =-\frac{1}{\sqrt{2}} \\
H_{x_{2} y_{2}} & =0 \\
H_{x_{3} y_{3}} & =\frac{1}{\sqrt{2}}\end{aligned}$ \\
\hline$N_{8}=2$ & $\begin{array}{c}\left(m_{x_{1} y_{1}}, n_{x_{1} y_{1}}\right)=(-1,1) \\
\left(m_{x_{2} y_{2}}, n_{x_{2} y_{2}}\right)=(1,1) \\
\left(m_{x_{3} y_{3}}, n_{x_{3} y_{3}}\right)=(-1,1)\end{array}$ & $\begin{aligned} H_{x_{1} y_{1}} & =-\frac{1}{2 \sqrt{2}} \\
H_{x_{2} y_{2}} & =\sqrt{2} \\
H_{x_{3} y_{3}} & =-\frac{1}{\sqrt{2}}\end{aligned}$ \\
\hline$N_{9}=1$ & $\begin{array}{c}\left(m_{x_{1} y_{1}}, n_{x_{1} y_{1}}\right)=(0,1) \\
\left(m_{x_{2} y_{2}}, n_{x_{2} y_{2}}\right)=(-1,1) \\
\left(m_{x_{3} y_{3}}, n_{x_{3} y_{3}}\right)=(2,1)\end{array}$ & $\begin{array}{l}H_{x_{1} y_{1}}=0 \\
H_{x_{2} y_{2}}=-\sqrt{2} \\
H_{x_{3} y_{3}}=\sqrt{2}\end{array}$ \\
\hline$N_{0}=3$ & $\left(m_{i j}, n_{i j}\right)=(0, \pm 1)$ & $H_{i j}=0$ \\
\hline
\end{tabular}


Notice that all wrapping numbers are equal to \pm 1 .

The first six stacks of magnetized branes, each preserving $\mathcal{N}=2$ susy, share a common $\mathcal{N}=1$ susy. The supersymmetry conditions in (??) fix the nine deformations (6 NS-NS, 3 R-R) of the complex structure to be diagonal and purely imaginary, $\tau_{I J}=i \delta_{I J}$. In addition there are three stacks of magnetized branes, two of which preserve $\mathcal{N}=2$ susy, while the remaining one preserves the $\mathcal{N}=1$ susy shared by all the branes. The stability conditions, trivially satisfied by the first six stacks, fix the Kähler structure when imposed on the latter three stacks.

The resulting Kähler form reads $J=J_{(1)}+J_{(2)}+J_{(3)}$ with

$$
\begin{aligned}
& J_{(1)}=4 \pi^{2} 2 \sqrt{2} \alpha^{\prime} \frac{d z_{1} \wedge d \overline{z_{1}}}{2 i} \\
& J_{(2)}=\frac{4 \pi^{2}}{\sqrt{2}} \alpha^{\prime} \frac{d z_{2} \wedge d \overline{z_{2}}}{2 i} \\
& J_{(3)}=4 \pi^{2} \sqrt{2} \alpha^{\prime} \frac{d z_{3} \wedge d \overline{z_{3}}}{2 i}
\end{aligned}
$$

i.e. radii $r_{1}=r_{2}=2^{3 / 4} \sqrt{\alpha^{\prime}}, r_{3}=r_{4}=2^{-1 / 4} \sqrt{\alpha^{\prime}}, r_{5}=r_{6}=2^{1 / 4} \sqrt{\alpha^{\prime}}$. With the above supersymmetric choice of fluxes, we computed the ortogonal $6 \times 6$ matrix, $R_{a}$, for each stack of branes. They read

$$
\begin{gathered}
R_{1}^{ \pm 1}=\left(\begin{array}{ccc}
-\frac{1}{3} \mathbb{1} & \mp \frac{2 \sqrt{2}}{3} i \sigma_{2} & 0 \\
\mp \frac{2 \sqrt{2}}{3} i \sigma_{2} & -\frac{1}{3} \mathbb{1} & 0 \\
0 & 0 & \mathbb{1}
\end{array}\right) \quad R_{3}^{ \pm 1}=\left(\begin{array}{ccc}
-\frac{1}{3} \mathbb{1} & \mp \frac{2 \sqrt{2}}{3} \mathbb{1} & 0 \\
\pm \frac{2 \sqrt{2}}{3} \mathbb{1} & -\frac{1}{3} \mathbb{1} & 0 \\
0 & 0 & \mathbb{1}
\end{array}\right) \\
R_{2}^{ \pm 1}=\left(\begin{array}{ccc}
-\frac{3}{5} \mathbb{1} & 0 & \pm \frac{4}{5} i \sigma_{2} \\
0 & \mathbb{1} & 0 \\
\pm \frac{4}{5} i \sigma_{2} & 0 & -\frac{3}{5} \mathbb{1}
\end{array}\right) \quad R_{5}^{ \pm 1}=\left(\begin{array}{ccc}
-\frac{3}{5} \mathbb{1} & 0 & \pm \frac{4}{5} \mathbb{1} \\
0 & \mathbb{1} & 0 \\
\mp \frac{4}{5} \mathbb{1} & 0 & -\frac{3}{5} \mathbb{1}
\end{array}\right) \\
R_{4}^{ \pm 1}=\left(\begin{array}{ccc}
\mathbb{1} & 0 & 0 \\
0 & 0 & \mp \mathbb{1} \\
0 & \pm \mathbb{1} & 0
\end{array}\right) \quad R_{6}^{ \pm 1}=\left(\begin{array}{ccc}
\mathbb{1} & 0 & 0 \\
0 & 0 & \pm i \sigma_{2} \\
0 & \pm i \sigma_{2} & 0
\end{array}\right) \\
R_{7}^{ \pm 1}=\left(\begin{array}{ccc}
\frac{1}{3} \mathbb{1} \pm \frac{2 \sqrt{2}}{3} i \sigma_{2} & 0 \\
0 & \mathbb{1} \\
0 & 0 & \frac{1}{3} \mathbb{1} \mp \frac{2 \sqrt{2}}{3} i \sigma_{2}
\end{array}\right)
\end{gathered}
$$




$$
\begin{gathered}
R_{8}^{ \pm 1}=\left(\begin{array}{ccc}
\frac{7}{9} \mathbb{1} \pm \frac{4 \sqrt{2}}{9} i \sigma_{2} & 0 & 0 \\
0 & -\frac{1}{3} \mathbb{1} \mp \frac{2 \sqrt{2}}{3} i \sigma_{2} & 0 \\
0 & 0 & \frac{1}{3} \mathbb{1} \pm \frac{2 \sqrt{2}}{3} i \sigma_{2}
\end{array}\right) \\
R_{9}^{ \pm 1}=\left(\begin{array}{ccc}
\mathbb{1} & 0 & 0 \\
0 & -\frac{1}{3} \mathbb{1} \pm \frac{2 \sqrt{2}}{3} i \sigma_{2} & 0 \\
0 & 0 & -\frac{1}{3} \mathbb{1} \mp \frac{2 \sqrt{2}}{3} i \sigma_{2}
\end{array}\right)
\end{gathered}
$$

Switching to complex coordinates $z^{i}=x^{i}+i y^{i}$ the above matrices become block diagonal

$$
R_{a}^{ \pm 1}=\left(\begin{array}{cc}
U_{a}^{ \pm 1} & 0 \\
0 & U_{a}^{* \pm 1}
\end{array}\right)
$$

where the sub-blocks $U_{a}$ are $3 \times 3$ complex matrices belonging to $S U(3) \subset$ $S U(4)$. They read

$$
\begin{aligned}
& U_{1}^{ \pm 1}=\left(\begin{array}{ccc}
-\frac{1}{3} & \mp \frac{2 \sqrt{2}}{3} i & 0 \\
\mp \frac{2 \sqrt{2}}{3} i & -\frac{1}{3} & 0 \\
0 & 0 & 1
\end{array}\right) \quad U_{3}^{ \pm 1}=\left(\begin{array}{ccc}
-\frac{1}{3} & \pm \frac{2 \sqrt{2}}{3} & 0 \\
\mp \frac{2 \sqrt{2}}{3} & -\frac{1}{3} & 0 \\
0 & 0 & 1
\end{array}\right) \\
& U_{2}^{ \pm 1}=\left(\begin{array}{ccc}
-\frac{3}{5} & 0 & \mp \frac{4}{5} i \\
0 & 1 & 0 \\
\mp \frac{4}{5} i & 0 & -\frac{3}{5}
\end{array}\right) \quad U_{5}^{ \pm 1}=\left(\begin{array}{ccc}
-\frac{3}{5} & 0 & \pm \frac{4}{5} \\
0 & 1 & 0 \\
\mp \frac{4}{5} & 0 & -\frac{3}{5}
\end{array}\right) \\
& U_{4}^{ \pm 1}=\left(\begin{array}{ccc}
1 & 0 & 0 \\
0 & 0 & \pm 1 \\
0 & \mp 1 & 0
\end{array}\right) \quad U_{6}^{ \pm 1}=\left(\begin{array}{ccc}
1 & 0 & 0 \\
0 & 0 & \mp i \\
0 & \mp i & 0
\end{array}\right) \\
& U_{7}^{ \pm 1}=\left(\begin{array}{ccc}
\frac{1}{3} \mp \frac{2 \sqrt{2}}{3} i & 0 & 0 \\
0 & 1 & 0 \\
0 & 0 & \frac{1}{3} \pm \frac{2 \sqrt{2}}{3} i
\end{array}\right)
\end{aligned}
$$




$$
\begin{gathered}
U_{8}^{ \pm 1}=\left(\begin{array}{ccc}
\frac{7}{9} \mp \frac{4 \sqrt{2}}{9} i & 0 & 0 \\
0 & -\frac{1}{3} \pm \frac{2 \sqrt{2}}{3} i & 0 \\
0 & 0 & \frac{1}{3} \mp \frac{2 \sqrt{2}}{3} i
\end{array}\right) \\
U_{9}^{ \pm 1}=\left(\begin{array}{ccc}
1 & 0 & 0 \\
0 & -\frac{1}{3} \mp \frac{2 \sqrt{2}}{3} i & 0 \\
0 & 0 & -\frac{1}{3} \pm \frac{2 \sqrt{2}}{3} i
\end{array}\right)
\end{gathered}
$$

Except for the stacks labelled by $a=8$, all the submatrices have, at least, one unit eigenvalue (in fact three for $a=0$ ). The associated eigenvector defines a (complex) unmagnetized direction with standard KK momenta. The other two eigenvalues are complex conjugate.

As usual the open string states can be grouped into three main sectors: neutral, singly and doubly charged ones. With an abuse of notation we will often call magnetic shifts the angles $\beta=\pi \epsilon$.

The uncharged sector comprise open strings stretching either between two unmagnetized branes and carrying standard KK momenta or strings starting and ending on the same stack of magnetized branes $\left(q_{a}=-q_{b}\right)$ and carrying reshuffled KK momenta and windings.

In the singly charged sector, one endpoint of the string is on the $N_{0}$ stack, the other is on one of the stack of magnetized branes, $N_{a}$, or their images under $\Omega, N_{a}^{*}$. The frequencies of the oscillator modes are shifted by

$$
\beta_{0 a}^{(k)}=\frac{\arg \left(\rho_{a}^{(k)}\right)}{2 \pi}
$$

Open strings in this sector appear with a multiplicity due to the degeneracy of the Landau levels. For $a=8$, this is given by the product of the magnetic numbers $\prod_{I} m_{a}^{I}$, in all other cases it is reduced to $\prod_{I \neq u} m_{a}^{I}$ for the presence of some unmagnetized plane, generically indicated by $u$.

Charged strings, starting on magnetized stack and arriving at its images are doubly charged $\left(q_{a}=q_{b}= \pm 1\right)$. The frequencies shift is

$$
2 \beta_{a a}^{(k)}=\frac{\arg \left(\rho_{a}^{(k)}\right)}{\pi}
$$

and the multiplicities are $\prod_{I}\left(2 m_{8}^{I}\right)$ for $a=8$ or $\prod_{I \neq u}\left(2 m_{a}^{I}\right)$ for all $a \neq 8$.

The situation becomes more involved when strings, starting on magnetized stack of branes (say, $N_{a}$ ) and ending on a different stack (labelled by $N_{b}^{*}$, or its image ), are taken into account. In all, there are 36 distinct [a,b] sectors. 
In the three sectors $[1,3],[2,5],[4,6]$ diagonalization of $R_{a}^{q_{a}} R_{b}^{q_{b}}$ yields one $\rho_{a b}^{u}=1$ and two complex conjugate pairs determined by

$$
\tan \left(2 \beta_{a b}^{I}\right)= \pm \sqrt{t_{a}^{2}+t_{b}^{2}+t_{a}^{2} t_{b}^{2}} \quad \text { for } I \neq u
$$

where $t_{a / b}=\tan \left(\beta_{a / b}^{I}\right)$.

In the 3 sectors $[7,8],[7,9],[8,9]$, due to diagonal form of the submatrices $U_{i}^{q_{a}}$, the magnetic shifts are simply

$$
\beta_{a b}^{I}= \pm \beta_{a}^{I} \pm \beta_{b}^{I}
$$

and there is no unmagnetized direction.

The twelve sectors [13]-[25], [25]-[46] and [46]-[13] all have one unit eigenvalue (one complex unmagnetized direction/plane) and a complex conjugate pair, $\rho_{a b}^{ \pm}$. More explicitly one finds

$$
\begin{aligned}
& \rho_{[1,3]-[2,5]}^{ \pm}=-\frac{1}{15}(13 \mp 2 \sqrt{14}) \\
& \rho_{[2,5]-[4,6]}^{ \pm}=-\frac{4}{5} \pm \frac{3 i}{5} \\
& \rho_{[4,6]-[1,3]}^{ \pm}=-\frac{2}{3} \pm \frac{i \sqrt{5}}{3} .
\end{aligned}
$$

The remaining 18 sectors given by [789]-[123456] can have common unmagnetized planes and frequencies given by

$$
\begin{aligned}
\beta_{L a b}^{I} & =\beta_{L a}^{I} \\
\tan \left(2 \beta_{R a b}^{I}\right) & = \pm \sqrt{t_{a R}^{2}+t_{b R}^{2}+t_{a R}^{2} t_{b a R}^{2}}
\end{aligned}
$$

for $I \neq u$. The results are listed in the following tables. 


\begin{tabular}{|l|c|l|}
\hline sectors & \multicolumn{1}{|c|}{ eigenvalues } & unmagnetized directions \\
\hline$U_{4}^{q_{a}} U_{1}^{q_{b}}$ & $\rho=1$ and $\rho^{ \pm}=(-2 \pm i \sqrt{5}) / 3$ & $\left(-\frac{i q_{a} q_{b}}{\sqrt{2}}, q_{a}, 1\right)$ \\
\hline$U_{4}^{q_{a}} U_{3}^{q_{b}}$ & $\rho=1$ and $\rho^{ \pm}=(-2 \pm i \sqrt{5}) / 3$ & $\left(\frac{q_{a} q_{b}}{\sqrt{2}}, q_{a}, 1\right)$ \\
\hline$U_{6}^{q_{a}} U_{1}^{q_{b}}$ & $\rho=1$ and $\rho^{ \pm}=(-2 \pm i \sqrt{5}) / 3$ & $\left(-\frac{q_{a} q_{b}}{\sqrt{2}},-i q_{a}, 1\right)$ \\
\hline$U_{6}^{q_{a}} U_{3}^{q_{b}}$ & $\rho=1$ and $\rho^{ \pm}=(-2 \pm i \sqrt{5}) / 3$ & $\left(-\frac{i q_{a} q_{b}}{\sqrt{2}},-q_{a}, 1\right)$ \\
\hline$U_{2}^{q_{a}} U_{4}^{q_{b}}$ & $\rho=1$ and $\rho^{ \pm}=(-4 \pm i 3) / 5$ & $\left.\frac{i q_{a}}{2}, q_{b}, 1\right)$ \\
\hline$U_{2}^{q_{a}} U_{6}^{q_{b}}$ & $\rho=1$ and $\rho^{ \pm}=(-4 \pm i 3) / 5$ & $\left(\frac{i q_{a}}{2},-i q_{b}, 1\right)$ \\
\hline$U_{5}^{q_{a}} U_{4}^{q_{b}}$ & $\rho=1$ and $\rho^{ \pm}=(-4 \pm i 3) / 5$ & $\left(-\frac{q_{a}}{2}, q_{b}, 1\right)$ \\
\hline$U_{5}^{q_{a}} U_{6}^{q_{b}}$ & $\rho=1$ and $\rho^{ \pm}=(-4 \pm i 3) / 5$ & $\left(-\frac{q_{a}}{2},-i q_{b}, 1\right)$ \\
\hline$U_{1}^{q_{a}} U_{2}^{q_{b}}$ & $\rho=1$ and $\rho^{ \pm}=(-13 \pm \sqrt{56}) / 15$ & $\left(2 i q_{b},-\sqrt{2} q_{a} q_{b}, 1\right)$ \\
\hline$U_{1}^{q_{a}} U_{5}^{q_{b}}$ & $\rho=1$ and $\rho^{ \pm}=(-13 \pm \sqrt{56}) / 15$ & $\left(-2 q_{b},-i \sqrt{2} q_{a} q_{b}, 1\right)$ \\
\hline$U_{3}^{q_{a}} U_{2}^{q_{b}}$ & $\rho=1$ and $\rho^{ \pm}=(-13 \pm \sqrt{56}) / 15$ & $\left(2 i q_{b}, i \sqrt{2} q_{a} q_{b}, 1\right)$ \\
\hline$U_{3}^{q_{a}} U_{5}^{q_{b}}$ & $\rho=1$ and $\rho^{ \pm}=(-13 \pm \sqrt{56}) / 15$ & $\left(-2 q_{b},-\sqrt{2} q_{a} q_{b}, 1\right)$ \\
\hline
\end{tabular}

\begin{tabular}{|l|c|l|}
\hline sectors & eigenvalues & unmagnetized directions \\
\hline$U_{1}^{q_{a}} U_{3}^{q_{b}}$ & $\rho=1$ and $\rho^{ \pm}=\frac{1}{9}(1 \pm 4 i \sqrt{5})$ & $(0,0,1)$ \\
\hline$U_{2}^{q_{a}} U_{5}^{q_{b}}$ & $\rho=1$ and $\rho^{ \pm}=\frac{1}{25}(9 \pm 4 i \sqrt{34})$ & $(0,1,0)$ \\
\hline$U_{4}^{q_{a}} U_{6}^{q_{b}}$ & $\rho=1$ and $\rho^{ \pm}= \pm i$ & $(1,0,0)$ \\
\hline$U_{7}^{q_{a}} U_{8}^{q_{b}}$ & $\rho_{1}=-\left(-7+16 q_{a} q_{b}+2 \sqrt{2} i\left(2 q_{b}+7 q_{a}\right)\right) / 27$ & \\
& $\rho_{2}=\left(1+8 q_{a} q_{b}+2 \sqrt{2} i\left(q_{a}-q_{b}\right)\right) / 9$ & no unmagnetized directions \\
& $\rho_{3}=-\left(1-2 i \sqrt{2} q_{b}\right) / 3$ & \\
\hline$U_{7}^{q_{a}} U_{9}^{q_{b}}$ & $\rho_{1}=-\frac{1}{3}\left(1+2 i \sqrt{2} q_{b}\right)$ & \\
& $\rho_{2}=-\left(1+8 q_{a} q_{b}+2 \sqrt{2} i\left(q_{a}-q_{b}\right)\right) / 9$ & no unmagnetized directions \\
& $\rho_{3}=\frac{1}{3}\left(1-2 i \sqrt{2} q_{a}\right)$ & \\
\hline$U_{8}^{q_{a}} U_{9}^{q_{b}}$ & $\rho_{1}=\frac{1}{9}\left(7-4 i \sqrt{2} q_{a}\right)$ & no unmagnetized directions \\
& $\rho_{2}=\left(1+8 q_{a} q_{b}-2 \sqrt{2} i\left(q_{a}-q_{b}\right)\right) / 9$ & \\
\hline
\end{tabular}




\begin{tabular}{|c|c|c|}
\hline sectors & eigenvalues & unmagnetized directions \\
\hline$U_{7}^{q_{a}} U_{1}^{q_{b}}$ & $\begin{array}{c}\rho_{1}=\frac{1}{3}\left(1+2 i \sqrt{2} q_{a}\right) \\
\rho_{2,3}=\frac{1}{9}\left(-2+i \sqrt{2} q_{a} \pm 5 \sqrt{\left(-1+2 i \sqrt{2} q_{a}\right)}\right.\end{array}$ & no unmagnetized directions \\
\hline$U_{7}^{q_{a}} U_{2}^{q_{b}}$ & $\rho_{1}=1$ and $\rho_{2,3}=\frac{1}{15}(-3 \pm 6 i \sqrt{6}$ & $(0,1,0)$ \\
\hline$U_{7}^{q_{a}} U_{3}^{q_{b}}$ & $\begin{array}{c}\rho_{1}=\frac{1}{3}\left(1+2 i \sqrt{2} q_{a}\right) \\
\rho_{2,3}=\frac{1}{9}\left(-2+i \sqrt{2} q_{a} \pm 5 \sqrt{\left(-1+2 i \sqrt{2} q_{a}\right)}\right.\end{array}$ & no unmagnetized directions \\
\hline$U_{7}^{q_{a}} U_{4}^{q_{b}}$ & $\begin{array}{l}\rho_{1}=\frac{1}{3}\left(1-2 i \sqrt{2} q_{a}\right) \\
\rho_{2,3}= \pm \frac{\sqrt{-1-2 i \sqrt{2} q_{a}}}{\sqrt{3}}\end{array}$ & no unmagnetized directions \\
\hline$U_{7}^{q_{a}} U_{5}^{q_{b}}$ & $\rho_{1}=1, \rho_{2,3}=\frac{1}{15}(-3 \pm 6 i \sqrt{6})$ & $(0,1,0)$ \\
\hline$U_{7}^{q_{a}} U_{6}^{q_{b}}$ & $\begin{array}{c}\rho_{1}=\frac{1}{3}\left(1-2 i \sqrt{2} q_{a}\right. \\
\rho_{2,3}= \pm \frac{\sqrt{-1-2 i \sqrt{2} q_{a}}}{\sqrt{3}}\end{array}$ & no unmagnetized directions \\
\hline$U_{8}^{q_{a}} U_{1}^{q_{b}}$ & $\begin{array}{c}\rho_{1}=\frac{1-2 i \sqrt{2} q_{a}}{3} \\
\rho_{2,3}=\frac{1}{27}\left(-2-i\left(\sqrt{2} q_{a} \pm \sqrt{241}\right)\right)\end{array}$ & no unmagnetized directions \\
\hline$U_{8}^{q_{a}} U_{2}^{q_{b}}$ & $\begin{array}{c}\rho_{1}=-\frac{1-2 i \sqrt{2} q_{a}}{3} \\
\rho_{2,3}=\frac{1}{3}\left(-1+i \sqrt{2} q_{a} \pm \sqrt{\left(2+4 i \sqrt{2} q_{a}\right)}\right)\end{array}$ & no unmagnetized directions \\
\hline$U_{8}^{q_{a}} U_{3}^{q_{b}}$ & $\begin{array}{c}\rho_{1}=\frac{1-2 i \sqrt{2} q_{a}}{3} \\
\rho_{2,3}=\frac{1}{27}\left(-2-i \sqrt{2} q_{a} \pm \sqrt{\left(-241-482 i \sqrt{2} q_{a}\right)}\right)\end{array}$ & no unmagnetized directions \\
\hline$U_{8}^{q_{a}} U_{4}^{q_{b}}$ & $\begin{array}{c}\rho_{1}=\frac{1}{9}\left(7-4 i \sqrt{2} q_{a}\right) \\
\rho_{2,3}= \pm \frac{1}{3} \sqrt{(-7-4 i \sqrt{2})}\end{array}$ & no unmagnetized directions \\
\hline$U_{8}^{q_{a}} U_{5}^{q_{b}}$ & $\begin{array}{c}\rho_{1}=-\frac{1}{3}\left(1-2 \sqrt{2} i q_{a}\right) \\
\rho_{2,3}=\frac{1}{3}\left(-1+i \sqrt{2} \pm \sqrt{2+i 4 \sqrt{2} q_{a}}\right)\end{array}$ & no unmagnetized directions \\
\hline$U_{8}^{q_{a}} U_{6}^{q_{b}}$ & $\begin{array}{c}\rho_{1}=\frac{1}{9}\left(7-4 i \sqrt{2} i q_{a}\right) \\
\rho 2,3= \pm \frac{1}{3} \sqrt{\left(-7-4 i \sqrt{2} i q_{a}\right)}\end{array}$ & no unmagnetized directions \\
\hline$\left.U_{9}^{q_{a}} U_{1}^{q_{b}}\right)$ & $\begin{array}{c}\rho_{1}=-\frac{1}{3}\left(1-2 \sqrt{2} i q_{a}\right) \\
\rho_{2,3}=\frac{1}{9}\left(-1+i \sqrt{2} q_{a} \pm \sqrt{26} \sqrt{\left(1+4 \sqrt{2} i q_{a}\right)}\right.\end{array}$ & no unmagnetized directions \\
\hline$\overline{\left.U_{9}^{q_{a}} U_{2}^{q_{b}}\right)}$ & $\begin{array}{c}\rho_{1}=-\frac{1}{3}\left(1+2 \sqrt{2} i q_{a}\right) \\
\rho_{2,3}=\frac{1}{15}\left(-3-3 \sqrt{2} i q_{a} \pm \sqrt{6} \sqrt{\left(11-22 i \sqrt{2} q_{a}\right)}\right.\end{array}$ & no unmagnetized directions \\
\hline$U_{9}^{q_{a}} U_{3}^{q_{b}}$ & $\begin{array}{c}\rho_{1}=-\frac{1}{3}\left(1-2 \sqrt{2} i q_{a}\right) \\
\rho_{2,3}=\frac{1}{9}\left(-1+i \sqrt{2} q_{a} \pm \sqrt{26} \sqrt{\left(1+4 \sqrt{2} i q_{a}\right)}\right.\end{array}$ & no unmagnetized directions \\
\hline$\left.U_{9}^{q_{a}} U_{4}^{q_{b}}\right)$ & $\rho_{1}=1, \rho_{2,3}= \pm i$ & $(1,0,0)$ \\
\hline$\left.\overline{U_{9}^{q_{a}}} U_{5}^{q_{b}}\right)$ & $\begin{array}{c}\rho_{1}=-\frac{1}{3}\left(1+2 \sqrt{2} i q_{a}\right) \\
\rho_{2,3}=\frac{1}{15}\left(-3-3 \sqrt{2} i q_{a} \pm \sqrt{6} \sqrt{\left(11-22 i \sqrt{2} q_{a}\right)}\right.\end{array}$ & no unmagnetized directions \\
\hline$\overline{\left.U_{9}^{q_{a}} U_{6}^{q_{b}}\right)}$ & $\rho_{1}=1, \rho_{2,3}= \pm i$ & $(1,0,0)$ \\
\hline
\end{tabular}




\section{References}

[1] E. S. Fradkin and A. A. Tseytlin, Phys. Lett. B 158 (1985) 316;

[2] A. Abouelsaood, C. G. . Callan, C. R. Nappi and S. A. Yost, Nucl. Phys. B 280 (1987) 599.

[3] V. V. Nesterenko, Int. J. Mod. Phys. A 4 (1989) 2627.

[4] C. Bachas and M. Porrati, Phys. Lett. B 296, 77 (1992) arXiv:hep-th/9209032.

[5] C. Bachas, arXiv:hep-th/9503030.

[6] E. Dudas, Class. Quant. Grav. 17 (2000) R41 arXiv:hep-ph/0006190|.

[7] R. Blumenhagen, L. Goerlich, B. Kors and D. Lust, JHEP 0010 (2000) 006 arXiv:hep-th/0007024, Fortsch. Phys. 49 (2001) 591 arXiv:hep-th/0010198; R. Blumenhagen, B. Kors and D. Lust, JHEP 0102 (2001) 030 arXiv:hep-th/0012156. R. Blumenhagen, B. Kors, D. Lust and T. Ott, Nucl. Phys. B 616 (2001) 3 arXiv:hep-th/0107138; R. Blumenhagen, V. Braun, B. Kors and D. Lust, JHEP 0207 (2002) 026 arXiv:hep-th/0206038; R. Blumenhagen, L. Gorlich and T. Ott, JHEP 0301 (2003) 021 arXiv:hep-th/0211059 ; D. Lust and S. Stieberger, arXiv:hep-th/0302221.

[8] G. Aldazabal, S. Franco, L. E. Ibanez, R. Rabadan and A. M. Uranga, J. Math. Phys. 42 (2001) 3103 arXiv:hep-th/0011073, JHEP 0102 (2001) 047 arXiv:hep-ph/0011132; L. E. Ibanez, F. Marchesano and R. Rabadan, JHEP 0111 (2001) 002 arXiv:hep-th/0105155; D. Cremades, L. E. Ibanez and F. Marchesano, JHEP 0207 (2002) 009 arXiv:hep-th/0201205|, JHEP 0207 (2002) 022 arXiv:hep-th/0203160|, arXiv:hep-th/0205074 A. M. Uranga, arXiv:hep-th/0208014.

[9] M. Cvetic, G. Shiu and A. M. Uranga, Phys. Rev. Lett. 87 (2001) 201801 arXiv:hep-th/0107143; M. Cvetic, G. Shiu and A. M. Uranga, Nucl. Phys. B 615 (2001) 3 arXiv:hep-th/0107166. M. Cvetic, P. Langacker and G. Shiu, Phys. Rev. D 66 (2002) 066004 arXiv:hep-ph/0205252, Nucl. Phys. B 642 (2002) 139 arXiv:hep-th/0206115|; M. Cvetic, I. Papadimitriou and G. Shiu, arXiv:hep-th/0212177; M. Cvetic and I. Papadimitriou, arXiv:hep-th/0303083, arXiv:hep-th/0303197. 
[10] C. Kokorelis, JHEP 0208 (2002) 036 arXiv:hep-th/0206108, arXiv:hep-th/0207234, arXiv:hep-th/0209202, arXiv:hep-th/0210200 arXiv:hep-th/0212281.

[11] S. Forste, G. Honecker and R. Schreyer, Nucl. Phys. B 593 (2001) 127 arXiv:hep-th/0008250, JHEP 0106 (2001) 004 arXiv:hep-th/0105208.

[12] D. Bailin, G. V. Kraniotis and A. Love, arXiv:hep-th/0108127, Phys. Lett. B 530 (2002) 202 arXiv:hep-th/0108131,, arXiv:hep-th/0208103, JHEP 0302 (2003) 052 arXiv:hep-th/0212112.

[13] C. Angelantonj and A. Sagnotti, Phys. Rept. 371 (2002) 1 arXiv:hep-th/0204089.

[14] M. Larosa, arXiv:hep-th/0111187, arXiv:hep-th/0212109 G. Pradisi, arXiv:hep-th/0210088 M. Larosa and G. Pradisi, Nucl. Phys. B 667, 261 (2003) arXiv:hep-th/0305224. G. Pradisi, arXiv:hep-th/0310154.

[15] L. Gorlich, arXiv:hep-th/0401040

[16] E. Kiritsis, Fortsch. Phys. 52, 200 (2004) arXiv:hep-th/0310001.

[17] R. Blumenhagen, arXiv:hep-th/0412025.

[18] C. Kokorelis, arXiv:hep-th/0402087.

[19] A. M. Uranga, Class. Quant. Grav. 20, S373 (2003) arXiv:hep-th/0301032.

[20] I. Antoniadis and T. Maillard, arXiv:hep-th/0412008.

[21] R. Rabadan, Nucl. Phys. B 620, 152 (2002) arXiv:hep-th/0107036.

[22] M. Marino, R. Minasian, G. W. Moore and A. Strominger, JHEP 0001, 005 (2000) arXiv:hep-th/9911206.

[23] M. Li, Nucl. Phys. B 460 (1996) 351 arXiv:hep-th/9510161; M. R. Douglas, arXiv:hep-th/9512077; C. Schmidhuber, Nucl. Phys. B 467 (1996) 146 arXiv:hep-th/9601003; M. B. Green, J. A. Harvey and G. W. Moore, Class. Quant. Grav. 14 (1997) 47 arXiv:hep-th/9605033; J. Mourad, Nucl. Phys. B 512 (1998) 199 arXiv:hep-th/9709012|; Y. K. Cheung and Z. Yin, Nucl. Phys. B 517 (1998) 69 arXiv:hep-th/9710206|; R. Minasian and G. W. Moore, JHEP 9711 (1997) 002 arXiv:hep-th/9710230. 
[24] M. R. Garousi and R. C. Myers, Nucl. Phys. B 475, 193 (1996) arXiv:hep-th/9603194.

[25] A. Hashimoto and I. R. Klebanov, Nucl. Phys. Proc. Suppl. 55B, 118 (1997) arXiv:hep-th/9611214.

[26] V. A. Kostelecky, O. Lechtenfeld and S. Samuel, Nucl. Phys. B 298, 133 (1988).

[27] M. Bianchi and Y. S. Stanev, Nucl. Phys. B 523 (1998) 193 arXiv:hep-th/9711069.

[28] M. Bianchi, G. Pradisi and A. Sagnotti, Nucl. Phys. B 376 (1992) 365; M. Bianchi, Nucl. Phys. B 528 (1998) 73 arXiv:hep-th/9711201;

[29] E. Witten, JHEP 9802 (1998) 006 arXiv:hep-th/9712028.

[30] Z. Kakushadze, G. Shiu and S. H. Tye, Phys. Rev. D 58 (1998) 086001 arXiv:hep-th/9803141; C. Angelantonj, Nucl. Phys. B 566 (2000) 126 arXiv:hep-th/9908064.

[31] F. Marchesano and G. Shiu, Phys. Rev. D 71, 011701 (2005) arXiv:hep-th/0408059.

[32] F. Marchesano and G. Shiu, JHEP 0411, 041 (2004) arXiv:hep-th/0409132.

[33] E. Dudas and C. Timirgaziu, arXiv:hep-th/0502085

[34] P. Di Vecchia and A. Liccardo, NATO Adv. Study Inst. Ser. C. Math. Phys. Sci. 556, 1 (2000) arXiv:hep-th/9912161. P. Di Vecchia and A. Liccardo, arXiv:hep-th/9912275.

[35] M. Berkooz, M. R. Douglas and R. G. Leigh, Nucl. Phys. B 480 (1996) 265 arXiv:hep-th/9606139; V. Balasubramanian and R. G. Leigh, Phys. Rev. D 55 (1997) 6415 arXiv:hep-th/9611165|.

[36] N. Kaloper and R. C. Myers, JHEP 9905, 010 (1999) arXiv:hep-th/9901045.

[37] S. Kachru, M. B. Schulz and S. Trivedi, JHEP 0310, 007 (2003) arXiv:hep-th/0201028. 
[38] R. Blumenhagen, D. Lust and T. R. Taylor, arXiv:hep-th/0303016, J. F. Cascales and A. M. Uranga, arXiv:hep-th/0303024.

[39] C. Angelantonj, S. Ferrara and M. Trigiante, JHEP 0310 (2003) 015 arXiv:hep-th/0306185. C. Angelantonj, S. Ferrara and M. Trigiante, Phys. Lett. B 582 (2004) 263 |arXiv:hep-th/0310136|. G. Dall'Agata and S. Ferrara, arXiv:hep-th/0502066.

[40] J. Dai, R. G. Leigh and J. Polchinski, Mod. Phys. Lett. A 4, 2073 (1989). R. G. Leigh, Mod. Phys. Lett. A 4, 2767 (1989).

[41] J. F. Morales, C. A. Scrucca and M. Serone, Nucl. Phys. B 552, 291 (1999) arXiv:hep-th/9812071. C. A. Scrucca and M. Serone, Nucl. Phys. B 556, 197 (1999) arXiv:hep-th/9903145.

[42] I. Antoniadis, E. Dudas and A. Sagnotti, Phys. Lett. B 464, 38 (1999) arXiv:hep-th/9908023. S. Sugimoto, Prog. Theor. Phys. 102 (1999) 685 arXiv:hep-th/9905159.

[43] A. Sagnotti, Phys. Lett. B 294 (1992) 196 arXiv:hep-th/9210127.

[44] L. E. Ibanez, R. Rabadan and A. M. Uranga, Nucl. Phys. B 542 (1999) 112 arXiv:hep-th/9808139. G. Aldazabal, D. Badagnani, L. E. Ibanez and A. M. Uranga, JHEP 9906 (1999) 031 arXiv:hep-th/9904071.

[45] M. Bianchi and J. F. Morales, JHEP 0003, 030 (2000) arXiv:hep-th/0002149.

[46] M. Bianchi and A. Sagnotti, Phys. Lett. B 247 (1990) 517, Nucl. Phys. B 361 (1991) 519.

[47] M.Bianchi, Ph.D. Thesis, preprint ROM2F-92/13;

[48] C. Angelantonj, M. Bianchi, G. Pradisi, A. Sagnotti and Y. S. Stanev, Phys. Lett. B 385 (1996) 96 arXiv:hep-th/9606169.

[49] C. Angelantonj, M. Bianchi, G. Pradisi, A. Sagnotti and Y. S. Stanev, Phys. Lett. B 387, 743 (1996) arXiv:hep-th/9607229.

[50] M. Bianchi, J. F. Morales and G. Pradisi, Nucl. Phys. B 573 (2000) 314 arXiv:hep-th/9910228. 
[51] T. P. T. Dijkstra, L. R. Huiszoon and A. N. Schellekens, arXiv:hep-th/0403196. T. P. T. Dijkstra, L. R. Huiszoon and A. N. Schellekens, arXiv:hep-th/0411129.

[52] J. Scherk and J. H. Schwarz, Nucl. Phys. B 153 (1979) 61.

[53] R. Rohm, Nucl. Phys. B 237 (1984) 553; S. Ferrara, C. Kounnas and M. Porrati, Phys. Lett. B 181 (1986) 263, Nucl. Phys. B 304 (1988) 500. Phys. Lett. B 206 (1988) 25; C. Kounnas and M. Porrati, Nucl. Phys. B 310 (1988) 355. I. Antoniadis, C. Bachas, D. C. Lewellen and T. N. Tomaras, Phys. Lett. B 207 (1988) 441; S. Ferrara, C. Kounnas, M. Porrati and F. Zwirner, Nucl. Phys. B 318 (1989) 75; C. Kounnas and B. Rostand, Nucl. Phys. B 341 (1990) 641; I. Antoniadis, Phys. Lett. B 246 (1990) 377. I. Antoniadis and C. Kounnas, Phys. Lett. B 261 (1991) 369. E. Kiritsis and C. Kounnas, Nucl. Phys. B 503 (1997) 117 arXiv:hep-th/9703059 .

[54] I. Antoniadis, E. Dudas and A. Sagnotti, Nucl. Phys. B 544 (1999) 469 hep-th/9807011; I. Antoniadis, G. D'Appollonio, E. Dudas and A. Sagnotti, Nucl. Phys. B 553 (1999) 133 hep-th/9812118; R. Blumenhagen and L. Gorlich, Nucl. Phys. B 551 (1999) 601 hep-th/9812158; C. Angelantonj, I. Antoniadis and K. Forger, Nucl. Phys. B 555 (1999) 116 hep-th/9904092; A. L. Cotrone, Mod. Phys. Lett. A 14 (1999) 2487 arXiv:hep-th/9909116.

[55] A. Sagnotti, "Anomaly cancellations and open string theories," in Erice 1992, Proceedings, "From superstrings to supergravity," arXiv:hep-th/9302099

[56] M. Bianchi, F. Fucito, A. Lionetto, M. Prisco, E. Trevigne, work in progress.

[57] C. Angelantonj, M. Bianchi, G. Pradisi, A. Sagnotti, Ya. Stanev, work in fieri.

[58] R. Blumenhagen, M. Cvetic, F. Marchesano and G. Shiu, arXiv:hep-h/0502095.

[59] R. Blumenhagen, M. Cvetic, P. Langacker and G. Shiu, arXiv:hep-th/0502005.

[60] R. Blumenhagen, D. Lust and S. Stieberger, JHEP 0307, 036 (2003) arXiv:hep-th/0305146. D. Lust and S. Stieberger, arXiv:hep-th/0302221 
[61] M. Mihailescu, I. Y. Park and T. A. Tran, Phys. Rev. D 64, 046006 (2001) arXiv:hep-th/0011079.

[62] I. Antoniadis, S. Dimopoulos and G. R. Dvali, Nucl. Phys. B 516 (1998) 70 arXiv:hep-ph/9710204. I. Antoniadis, N. Arkani-Hamed, S. Dimopoulos and G. R. Dvali, Phys. Lett. B 436 (1998) 257 arXiv:hep-ph/9804398.

[63] D. Cremades, L. E. Ibanez and F. Marchesano, JHEP 0307, 038 (2003) arXiv:hep-th/0302105. D. Cremades, L. E. Ibanez and F. Marchesano, JHEP 0405, 079 (2004) arXiv:hep-th/0404229.

[64] S. A. Abel and B. W. Schofield, arXiv:hep-th/0412206. D. Lust, P. Mayr, R. Richter and S. Stieberger, Nucl. Phys. B 696, 205 (2004) arXiv:hep-th/0404134. 